\title{
Design of High Quantum Efficiency and High Resolution, Si/SiGe Avalanche Photodiode Focal Plane Arrays Using Novel, Back- Illuminated, Silicon-on-Sapphire Substrates
}

\author{
Alvin G. Stern \\ AG STERN, LLC, Newton, MA 02467
}

USA

\section{Introduction}

The design and development of large scale, high quantum efficiency and high resolution silicon and silicon-germanium ( $\mathrm{Si} / \mathrm{SiGe}$ ) avalanche photodiode (APD) focal plane arrays (FPAs) is an active topic of research due to the wide range of scientific, medical and industrial applications for such high sensitivity imagers. Avalanche photodiodes can attain single photon sensitive operation due the large internal device gain that compensates and can fully eliminate the electronic readout noise normally limiting the sensitivity of solidstate detector devices, hence their importance in electronic imaging. Large, wafer scale arrays of ultra sensitive, high resolution silicon and silicon-germanium avalanche photodiodes have not been developed yet, primarily due to the increased fabrication complexity of such detector devices and arrays compared to the more common, nonavalanching detectors such as CCDs and CMOS-APS devices. One major fabrication challenge for avalanche type detectors is the requirement of providing effective optical isolation between adjacent detectors in an array since the avalanche gain process produces photons that could create false detection events in neighboring pixels and thereby increase the noise. Providing effective optical crosstalk isolation becomes more difficult for higher resolution arrays. While it is common for CCD arrays to have a pixel pitch between 12-30 $\mu \mathrm{m}$ and for CMOS-APS devices to have pixel pitch below $10 \mu \mathrm{m}$, it becomes more challenging to architect arrays of avalanche photodiodes for example, having such a small pitch due to optical crosstalk. The second major fabrication challenge for linear mode avalanche type detectors, especially critical in arrays is the detector gain uniformity. Detector gain uniformity is a critical performance parameter since an increase in gain excess noise will make the detector arrays unsuitable for precision metrology applications. As solid-state avalanche detectors are made smaller, it becomes more difficult to control the gain excess noise due to smaller area multiplication regions where the effects from slight variations in doping profiles and electric fields produce greater gain variability compared to larger area detectors. 
In this chapter, design aspects of a novel, back-illuminated silicon-on-sapphire material system are presented and compared to present substrate technologies to illustrate the capability of the novel substrates in solving optical crosstalk and detector gain uniformity fabrication challenges for producing high quantum efficiency and high resolution wafer scale arrays of $\mathrm{Si} / \mathrm{SiGe} \mathrm{APD}$ detector arrays. The novel substrate design incorporates a single crystal, epitaxially grown aluminum nitride antireflective layer between sapphire and silicon to improve optical transmittance into the silicon from sapphire. A $\lambda / 4-\mathrm{MgF}_{2}$ antireflective layer deposited on the backside of the sapphire improves optical transmittance from the ambient into the sapphire. The high transmittance, back-illuminated silicon-(AlN)sapphire substrates represent an enabling technology for producing radiation tolerant, high resolution, wafer scale arrays of solid-state light detectors. (Stern \& Cole, 2008) The Si and SiGe solid-state avalanche photodiodes for example, could be produced in highly uniform wafer scale arrays by liquid crystallographic etching of mesa pixels due to sapphire acting as a natural etch stopping layer. Mesa detectors and arrays would retain high quantum efficiency and sensitive-area-fill-factor respectively, due to light focusing monolithic sapphire microlenses beneath each pixel. The space between mesa detectors could be filled with metal to form a low-resistance contact across the array and also block direct pixel-topixel optical crosstalk. The closely integrated monolithic sapphire microlenses also help to address detector gain uniformity by focusing optical k-vectors directly into the active multiplication region of the avalanche photodiodes, thereby helping to improve the gain uniformity of the detectors and arrays. Coupled with recent advances in dual linear and Geiger-mode avalanche detector design, the novel substrates will enable wide dynamic range focal plane arrays operating near room temperature, capable of imaging over the full range of natural illumination conditions from AM 0 in space to a cloudy moonless night. (Stern \& Cole, 2010)

The novel, back-illuminated silicon-on-(AlN)-sapphire substrates offer the possibility of solving the fabrication challenges currently limiting the low cost availability of highly sensitive, wide dynamic range $\mathrm{Si}$ and $\mathrm{SiGe}$ avalanche photodiode arrays, including direct pixel-to-pixel optical crosstalk and detector gain uniformity. There still exists however, the phenomenon of indirect optical crosstalk by multiple reflections in the finite thickness, 50 $\mu \mathrm{m}$ thick sapphire substrate. It will be shown through detailed calculations and analysis means that indirect optical crosstalk through the $50 \mu \mathrm{m}$ thick sapphire substrate although present, will not prevent high resolution, $27 \mu \mathrm{m}$ pixel pitch Si/SiGe APD detector arrays operating in the highest (Geiger-mode) gain regimes with low noise across the full $1024 \times 1024$ pixel FPA for a $f / \#=5.6$ optical system. This significant result confirms that the novel substrates will enable a new class of highly sensitive, solid-state, wide dynamic range, $\mathrm{Si} / \mathrm{SiGe}$ detector arrays.

\section{Technology of silicon avalanche photodiode focal plane arrays}

The present approaches to fabricating solid-state Si/SiGe avalanche photodiode (APD) arrays have been constrained by the less than optimal substrates available for fabricating such specialized light detector arrays. Two prevailing approaches have been used in fabricating such APD detector device arrays and both approaches borrow heavily from the fabrication and substrate technology used in more common CCD and CMOS-APS sensor arrays. The first approach shown in Fig. 1 is the simplest and uses conventional CMOS foundry processing for electronic circuits that is also ordinarily used to fabricate low cost, 
front-illuminated CMOS-APS sensor arrays, to fabricate front-illuminated avalanche photodiode arrays. The silicon APD focal plane array design approach in Fig. 1 is known as planar CMOS technology because the detector array is fabricated in the same silicon substrate as the integrated pixel control readout electronics. The planar CMOS approach is cost effective because new substrate technology is not needed and existing silicon IC fabrication technology can be leveraged. Planar CMOS technology has been adapted in novel ways for silicon APD arrays by researchers in Italy and Switzerland. (Charbon, 2008; Guerrieri et al., 2009; Niclass et al., 2005) The usual limitations for solid-state detector arrays apply in using the planar silicon CMOS approach including reduced quantum efficiency inherent for front-illuminated devices and less than optimal array sensitive-area-fill-factor due to the space taken up by the pixel electronics.

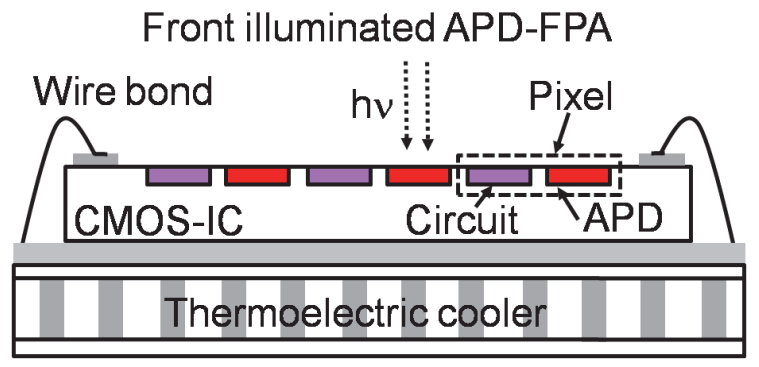

Fig. 1. Planar CMOS technology approach for fabricating cost effective silicon APD focal plane arrays.

\section{Back illuminated, hybrid APD-FPA}

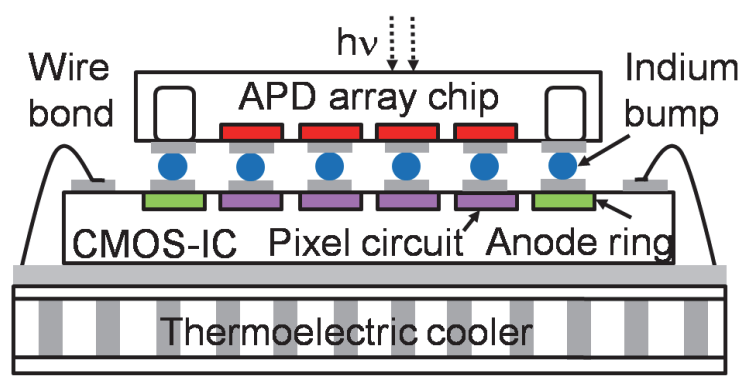

Fig. 2. Hybrid approach for fabricating high performance Si/SiGe APD focal plane arrays.

The second approach shown in Fig. 2, uses a hybridized focal plane array that consists of a back-illuminated detector array chip which is flip-chip bump-bonded or otherwise electrically mated to CMOS readout electronics. (Stern et al., 2003) The hybrid approach offers greater flexibility than the planar CMOS approach because the detector array can be designed in a different substrate material system from the CMOS control electronics. For example, the APD detector array could be fabricated from silicon, silicon-germanium, indium phosphide, indium gallium arsenide or mercury cadmium telluride. Moreover, back-illumination inherently supports higher detector quantum efficiency and array sensitive-area-fill-factor compared to 
front-illuminated planar arrays. The planar CMOS APD-FPA approach in Fig. 1 and the hybrid approach in Fig. 2 can both support integration of light focusing microlens arrays to increase the effective sensitive-area-fill-factor of the APD-FPAs, however, the planar CMOS approach is less amenable to microlens integration for the APDs since they would need to be epoxied to the CMOS chip and it is difficult to control epoxy thickness uniformity and refractive index matching. The hybrid APD-FPA approach however, supports microlenses to be monolithically integrated to the detectors without epoxy. The hybrid fabrication approach for silicon APD arrays has been implemented in the United States and is the preferred fabrication method resulting in higher performance arrays, albeit at increased cost. The hybrid approach shown in Fig. 2, has been used to fabricate focal plane arrays of silicon APD detectors using conventional silicon substrates that are back-thinned and either epoxied or oxide bonded to optically transparent quartz substrates followed by flip-chip bump-bonding to silicon CMOS readout ICs as shown in Figs. 3-4 respectively.

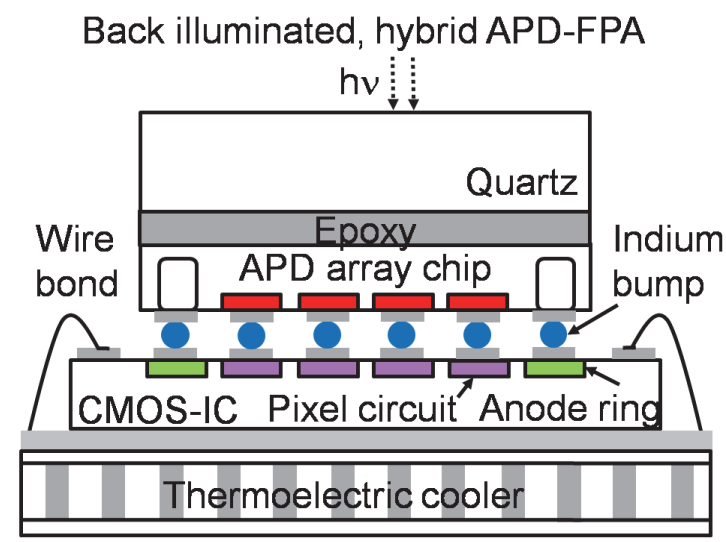

Fig. 3. Back-illuminated APD detector array silicon is thinned and epoxied to a quartz support wafer.

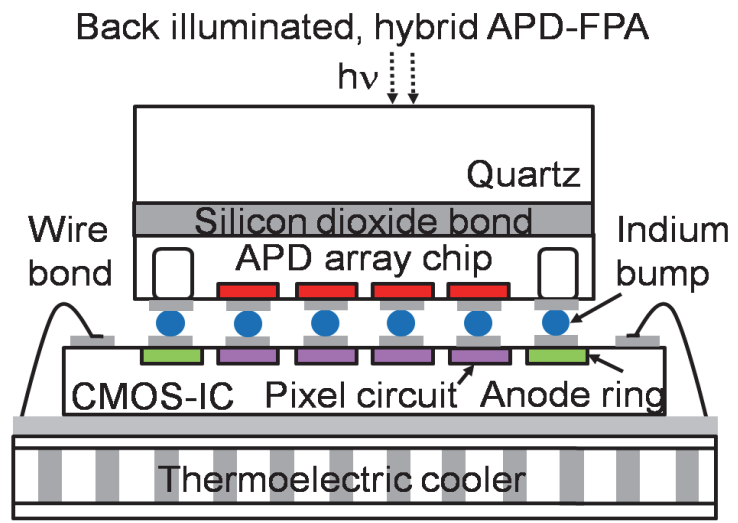

Fig. 4. Back-illuminated APD detector array silicon is thinned and oxide bonded to a quartz support wafer. 
The approaches for manufacturing hybrid Si/SiGe APD-FPAs shown in Figs. 3-4 are still non-optimal because the quartz substrate does not provide optimal light transmittance into the device silicon and also because quartz is not resistant to the common hydrofluoric acid (HF) etchant, used in silicon device processing. This may constrain silicon detector devices to be processed in the bulk silicon wafer prior to silicon thinning and subsequent oxide bonding or epoxying to the quartz substrate. As a result, the ultra sensitive detector devices might become damaged during the epoxying or oxide bonding process.

\subsection{Back-illuminated, silicon-on-sapphire substrates with improved antireflective layers for Si/SiGe APD-FPAs}

The silicon-on-sapphire material system is particularly well adapted for fabricating backilluminated, hybrid Si/SiGe APD-FPAs. Silicon-on-sapphire was discovered in 1963 by researchers working at the Boeing Corporation. Workers experimented with thermal decomposition of silane gas on a sapphire crystal polished into the shape of a sphere, thereby exposing all possible crystal planes, and discovered that (100) Si resulted from epitaxial growth on the R-plane surface of sapphire. (Manasevit \& Simpson, 1964) The advantages of (100) silicon-on-(R-plane)-sapphire (SOS) substrates soon became apparent in fabricating high speed, radiation resistant SOS-CMOS circuits for space electronics including the microprocessor of the Voyager I spacecraft launched in 1977. The problem of high defect densities due to lattice mismatch in the silicon close to the sapphire interface where FETs are fabricated, caused device reliability problems and kept integrated circuit production yields low. The resulting increased cost of production prevented the technology from gaining a wide market share for consumer electronics. In 1979, Lau discovered a method to improve the epitaxial growth of (100) silicon on R-plane sapphire, resulting in lower defect densities in the silicon near the sapphire interface. (Lau et al., 1979) In 1991, Imthurn developed a method of directly bonding a silicon wafer to the sapphire R-plane followed by thinning the silicon using chemical mechanical polishing to proper device thickness. He subsequently fabricated silicon test diodes that exhibited reverse dark currents one order of magnitude lower than similar devices fabricated in heteroepitaxially grown SOS. (Imthurn et al., 1992)

Although silicon-on-sapphire was originally developed for integrated circuit applications, it also has many ideal attributes for use as a substrate material, supporting back-illuminated, solid-state, $\mathrm{Si} / \mathrm{SiGe}$ detector arrays. Sapphire is an anisotropic, dielectric crystal of the negative uniaxial type that is weakly birefringent $\left(n_{\mathrm{o}}-n_{\mathrm{e}}=0.008\right)$ and possesses broadband optical transmittance ranging from the deep ultraviolet $\left(\lambda_{0}=200 \mathrm{~nm}\right)$ to the midwave IR $\left(\lambda_{0}\right.$ $=5500 \mathrm{~nm}$ ). Sapphire is extremely resilient, supporting thinning below $100 \mu \mathrm{m}$ which is an important requirement for high resolution, back-illuminated detector arrays. Sapphire can be optically polished to better than an 80-50 scratch and dig surface finish and can be etched using inductively coupled plasma (ICP) to fabricate light focusing microlenses beneath the silicon detectors. (Park et al., 2000) Sapphire is chemically resistant to most liquid etchants at room temperature and therefore functions as an ideal etchstop material during liquid crystallographic etching with tetramethyl ammonium hydroxide (TMAH) solution to define the silicon pixel mesa arrays. To enable high quantum efficiency, back-illuminated silicon detector arrays, the refractive index mismatch between air, sapphire and silicon has to be corrected however. The wide bandgap semiconductor material aluminum nitride (AIN), is closely lattice matched and refractive index matched to both sapphire and silicon and offers 
the prospect of enabling fabrication of high transmittance (100) silicon-on-(AIN)-sapphire substrates for back-illuminated silicon imagers. In 2008, Stern proposed introducing an epitaxially grown lattice matched and refractive index matched, single crystal aluminum nitride antireflective layer between the silicon and sapphire. (Stern \& Cole, 2008) The $\lambda / 4$ AIN antireflective layer helps to improve the back-illuminated optical transmittance from sapphire into the device silicon. A $\lambda / 4-\mathrm{MgF}_{2}$ antireflective layer can be deposited on the back surface of the thinned sapphire substrate to improve optical transmittance from the ambient into the sapphire. Figure 5 illustrates the back-illuminated silicon-on-sapphire substrate with $\lambda / 4$-AlN and $\lambda / 4-\mathrm{MgF}_{2}$ antireflective layers. Research shows that further improvement on the Si-(AlN)-sapphire substrate design from Fig. 5 can be achieved by incorporating an antireflective bilayer between sapphire and silicon, consisting of single crystal AlN and amorphous silicon nitride $(\mathrm{a}-\mathrm{SiN})$ as shown in Fig. 6. The design of the novel AlN/a-SiNx antireflective bilayer is analyzed in detail in Sec. 2.2.

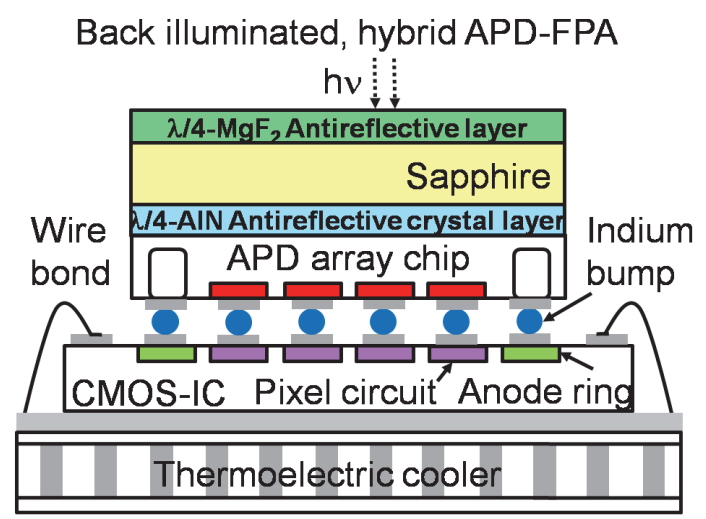

Fig. 5. Back-illuminated, hybrid, silicon-on-sapphire APD-FPA with $\lambda / 4-\mathrm{AlN}$ and $\lambda / 4-\mathrm{MgF}_{2}$ antireflective layers.

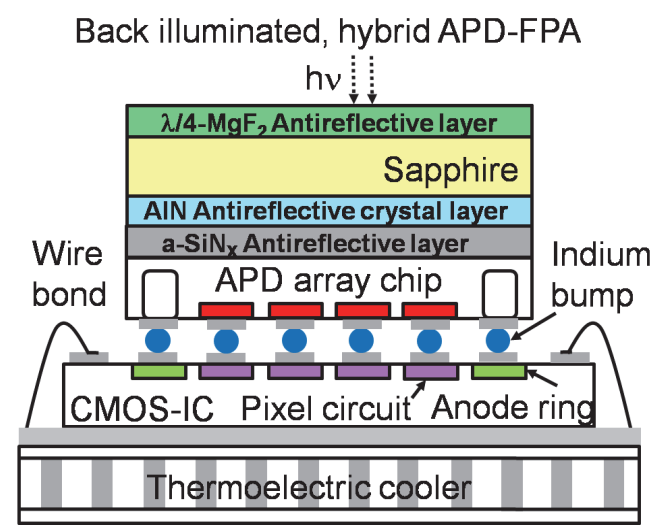

Fig. 6. Back-illuminated, hybrid, silicon-on-sapphire APD-FPA with AlN, $\mathrm{SiN}_{\mathrm{X}}$ and $\lambda / 4$ $\mathrm{MgF}_{2}$ antireflective layers. 
In contrast to silicon on quartz shown in Fig. 4, the Si-(AlN)-sapphire and Si-(AlN/a-SiNX)sapphire substrates shown in Figs. 5-6 can be prepared prior to detector device fabrication because the sapphire, $\mathrm{AlN}$ and a-SiN $\mathrm{x}$ material layers are not affected by hydrofluoric acid $(\mathrm{HF})$ or other etchants used in silicon device processing. Moreover, Si-(AlN/a-SiNx)sapphire substrates with $\lambda / 4-\mathrm{MgF}_{2}$ provide nearly optimal back-illuminated light transmittance into silicon as will be shown in Sec. 2.2, and in addition, microlenses can be directly fabricated in sapphire. (Park et al., 2000) Figure 7 shows a back-illuminated, crystallographically etched silicon mesa APD pixel with monolithically integrated sapphire microlens. Fig. 8 shows a crystallographically etched silicon mesa APD-FPA with monolithic, light focusing sapphire microlenses.

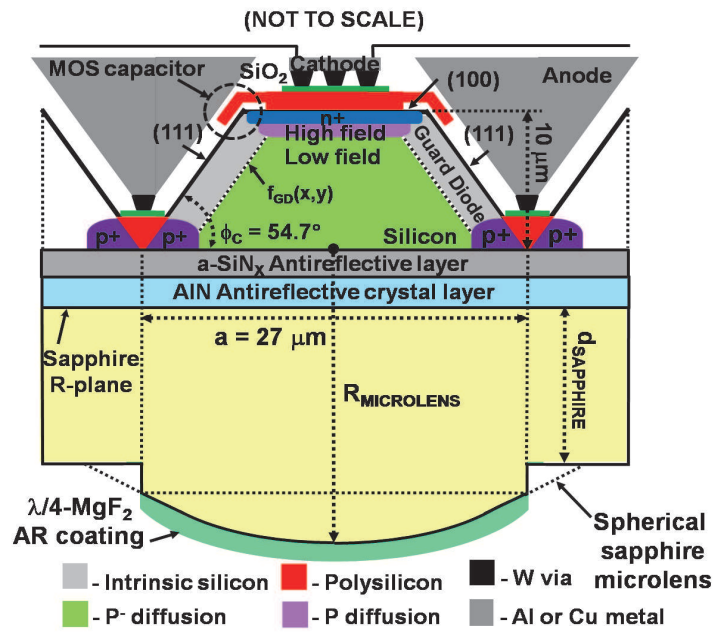

Fig. 7. Back-illuminated, silicon-on-sapphire mesa APD detector pixel with monolithic sapphire microlens.

Back illuminated, hybrid APD-FPA

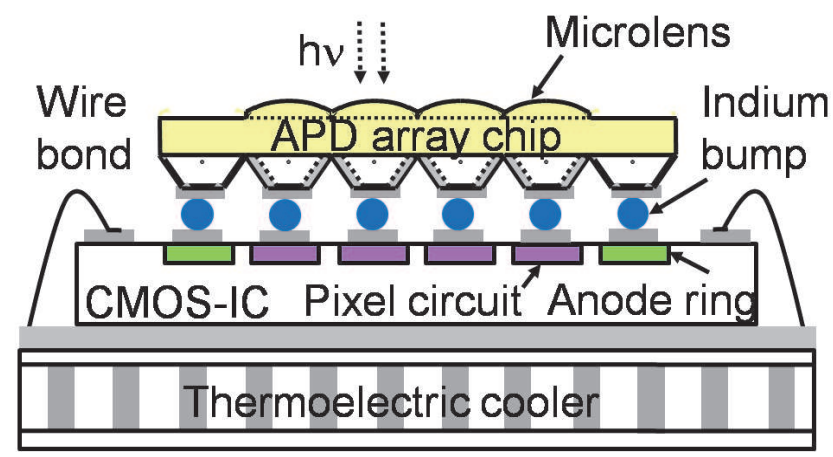

Fig. 8. Back-illuminated, hybrid, silicon-on-sapphire APD-FPA with monolithic sapphire microlenses. 
The sapphire substrate shown in Fig. 7 incorporates an antireflective bilayer between sapphire and silicon consisting of single crystal AIN and amorphous or a-SiN to improve optical transmittance into the device silicon. The space between mesa APD detector pixels is filled by a low resistance $\mathrm{Al}$ or $\mathrm{Cu}$ metal anode grid that provides low resistance anode contact at the base of each device mesa and also functions to block direct pixel-to-pixel optical crosstalk by line of sight light propagation. The monolithic sapphire microlens aligned beneath the mesa APD focuses light under the full height of the silicon mesa and away from the reduced height sidewalls. (Stern \& Cole, 2008)

\subsection{Advanced, very high transmittance silicon-on-sapphire substrate design for Si/SiGe APD-FPAs}

A variation on the back-illuminated $\mathrm{Si}$-(AlN)-sapphire substrate described in Sec. 2.1, provides improved optical transmittance into the device silicon by using an advanced antireflective bilayer design between sapphire and silicon consisting of single crystal AlN and non-stoichiometric, silicon rich, amorphous $\left(\mathrm{a}-\mathrm{SiN}_{\mathrm{x}}\right)$ with $\mathrm{x}<1.33$ as shown in Fig. 6 .

Stoichiometric, fully dense, silicon nitride $\left(\mathrm{Si}_{3} \mathrm{~N}_{4}\right.$ or $\left.\mathrm{SiN}_{1.33}\right)$ is an amorphous dielectric having a high optical bandgap, $E_{\mathrm{g}}=5.3 \mathrm{eV}$ and low optical absorption coefficient from UV to infrared. (Sze, 1981) Amorphous silicon nitride or a-SiNx thin films have many applications in silicon processing and device fabrication including surface and bulk passivation of silicon, antireflective layers for silicon solar cells, barrier layers against $\mathrm{Na}$ and $\mathrm{K}$ ion diffusion and CMOS transistor device isolation using the LOCOS method. (Plummer et al., 2000) In addition, silicon rich a-SiN $\mathrm{X}_{X<1.33}$ that has a higher refractive index and lower tensile strain than stoichiometric a- $\mathrm{SiN}_{1.33}$ has important applications for high speed optical interconnects in silicon nanophotonics and for silicon micromachining in MEMS and MOEMS applications. (Gardeniers at al., 1996)

Due to the ubiquity and importance of a-SiN $\mathrm{N}_{\mathrm{X}}$ thin films, much effort has been expended in developing optimized, application specific deposition methods for such films. Deposition of a-SiN $\mathrm{N}_{\mathrm{X}}$ is most readily achieved using low pressure $(<1 \mathrm{Atm}$.) gaseous precursors reacting either at low or high temperatures. High temperature, stoichiometric a- $\mathrm{SiN}_{1.33}$ films are most commonly deposited on substrates in a low pressure chemical vapor deposition (LPCVD) reactor according to the chemical reaction in Eq. (1). (Rosler, 1977)

$$
\begin{gathered}
3 \mathrm{SiH}_{2} \mathrm{Cl}_{2}+4 \mathrm{NH}_{3} \rightarrow \mathrm{Si}_{3} \mathrm{~N}_{4}+6 \mathrm{HCl}+6 \mathrm{H}_{2}+2 \mathrm{~N}_{2} \quad\left(700-900^{\circ} \mathrm{C}\right) \\
3 \mathrm{SiH}_{4}+4 \mathrm{NH}_{3} \rightarrow \mathrm{Si}_{3} \mathrm{~N}_{4}+12 \mathrm{H}_{2} \quad\left(700-900{ }^{\circ} \mathrm{C}\right)
\end{gathered}
$$

In Eq. (1), dichlorosilane (DCS) is shown as the silicon containing reactant species however, silane $\left(\mathrm{SiH}_{4}\right)$ can also be used as shown in Eq. (2). The advantage of DCS over silane is that the $\mathrm{HCl}$ byproduct can help remove metallic impurities from substrate surfaces by reacting to form volatile metal halides. Recently, much effort has been placed in developing low substrate temperature a-SiN $\mathrm{N}_{\mathrm{X}}$ deposition methods using plasma enhanced chemical vapor deposition (PECVD) and hot filament chemical vapor deposition (HFCVD), as such methods can be used to conserve valuable thermal budget during silicon device processing. In PECVD, a plasma reactor is used to enhance the chemical deposition while allowing substrate temperatures to remain in the low $200-450{ }^{\circ} \mathrm{C}$ temperature range. (Lowe et al., 1986) In HFCVD, an energized tungsten or tantalum filament heats the reactant gases while allowing low substrate temperatures to be used. (Verlaan et al., 2007) 
For the present application however, conservation of thermal budget is not a concern because the $\mathrm{Si}-\left(\mathrm{AlN} / \mathrm{a}-\mathrm{SiN}_{\mathrm{X}}\right)$-sapphire substrate can be fabricated before the mesa APD detector device. The a-SiN $x$ antireflective layer shown in Fig. 6, can be fabricated by direct deposition using LPCVD at elevated temperature, on a full thickness (100) silicon wafer according to the chemical reaction in Eq. (1). The sought after a-SiN $\mathrm{N}_{\mathrm{X}}$ antireflective layer characteristics listed in order from greatest to least in importance include, (1) refractive index, (2) optical bandgap $E_{g}$, (3) tensile strain in the layer and (4) surface and bulk passivation properties for silicon. Each of the four characteristics of the a-SiN $\mathrm{x}$ antireflective layer will be analyzed and/or discussed in order of importance for the present application. The primary role of the a-SiN $\mathrm{S}_{\mathrm{X}}$ is to function as an antireflective layer in conjunction with $\mathrm{AIN}$ as shown in Fig. 6, therefore, it is critical to design the layer to have a refractive index meeting the condition, $\mathrm{n}_{\mathrm{a}-\mathrm{SiN}}=\left(\mathrm{n}_{\mathrm{AIN}} \cdot \mathrm{n}_{\mathrm{Si}}\right)^{0.5}$ over most of the wavelength range of interest, to yield maximum optical transmittance from sapphire into the device silicon. The Sellmeier dispersion relation for stoichiometric a-SiN $\mathrm{N}_{1.33}$ is given in Eq. (3), with constants for the equation listed in Table 1.

$$
n^{2}(\lambda)=1+\frac{A_{1} \lambda^{2}}{\lambda^{2}-\lambda_{1}^{2}} \rightarrow(\lambda \text { in nm })
$$

Parame
$A_{1}$
$\lambda_{1}$
Value

2.8939

$139.67 \times 10^{-3}$

Table 1. Sellmeier dispersion relation constants for stoichiometric a-SiN $\mathrm{N}_{1.33}$ from Eq. (3).

The real refractive index of a-SiN $\mathrm{N}_{1.33}$ is plotted in Fig. 9 according to Eq. (3), using the parameters in Table 1. The real refractive indices for $\mathrm{MgF}_{2}$, sapphire, AlN and Si are also plotted for reference.

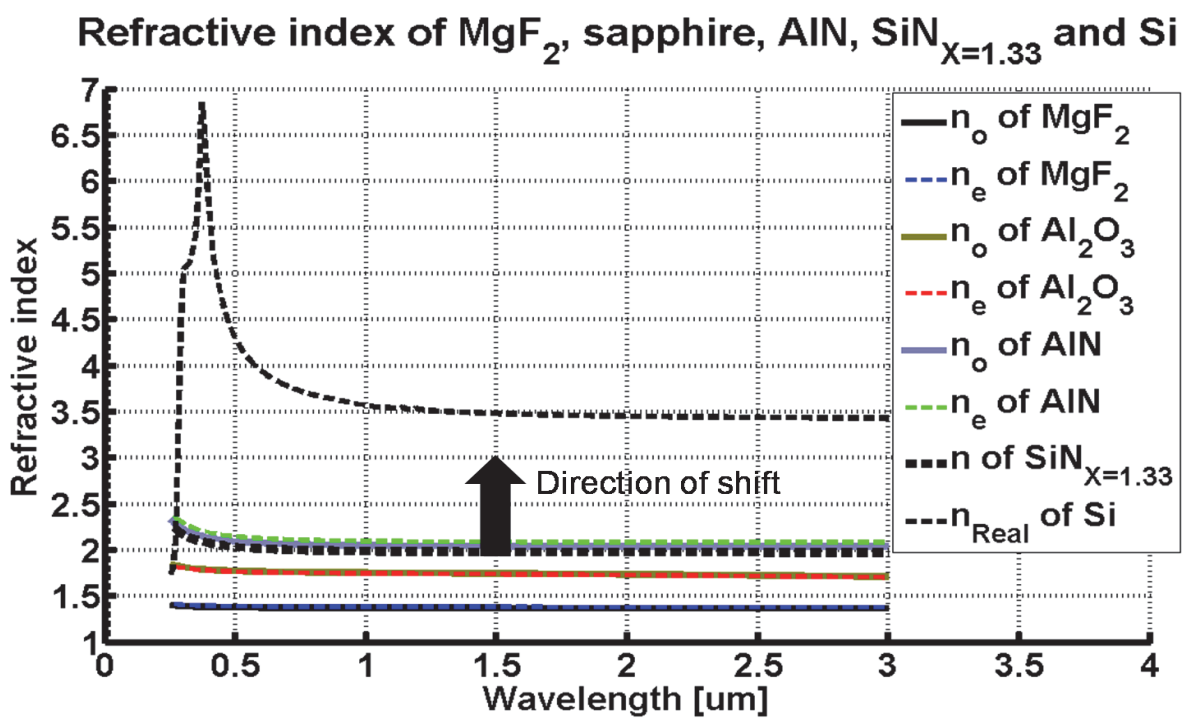

Fig. 9. Real refractive indices for $\mathrm{MgF}_{2}$, sapphire, $\mathrm{AlN}$, stoichiometric a-SiN $\mathrm{S}_{1.33}$ and $\mathrm{Si}$ are shown as a function of optical wavelength. 
The Sellmeier relation given by Eq. (3) and plotted in Fig. 9, shows that stoichiometric a$\mathrm{SiN}_{1.33}$ has a refractive index that is too low to provide refractive index matching between sapphire and silicon in conjunction with AIN as shown in Fig. 6, and the arrow in Fig. 9, represents the direction of vertical shift of the refractive index as a function of wavelength curve that would be required to provide refractive index matching. The Si content in a-SiN $\mathrm{N}_{1.33}$ therefore should be raised $(\mathrm{x}<1.33)$, to increase the refractive index of the layer, by increasing the DCS: $\mathrm{NH}_{3}$ gas flow ratio in Eq. (1). (Gardeniers et al., 1996)

The statistical experiments performed by Gardeniers, studied the properties of silicon rich a-SiN $\mathrm{X}_{<1.33}$ films deposited according to Eq. (1), by varying the primary process parameters including (1) temperature, (2) total pressure, (3) total gas flow, (4) DCS: $\mathrm{NH}_{3}$ gas flow ratio. Although their goal was to optimize the a-SiN $\mathrm{S}_{\mathrm{X}}$ thin films for micromechanical or MEMS applications requiring low tensile strain, their results also confirmed an important theoretical model described by Makino, predicting the a-SiN thin film refractive index as a function of the nitrogen to silicon ratio $(x=\mathrm{N}: \mathrm{Si})$ in the film. The model assumes that the refractive index of a-SiN $\mathrm{X}_{x \leq 1.33}$ films is a "bonddensity-weighted linear combination" of a-Si and a-SiN $\mathrm{S}_{1.33}$ reference refractive indices and is given by Eq. (4). (Makino, 1983)

$$
n=\frac{[(4 / x)-3] n_{0}+6 n_{1.33}}{(4 / x)+3}
$$

In Eq. (4), the quantity $n_{0}$ represents the refractive index of a-Si and $n_{1.33}$ represents the refractive index of stoichiometric a-SiN $\mathrm{S}_{1.33}$. Although the refractive index model in Eq. (4) does not consider the presence of residual hydrogen in the a-SiN $\mathrm{N}_{\mathrm{X}}$ thin film in the form of $\mathrm{Si}$ $\mathrm{H}$ and $\mathrm{N}-\mathrm{H}$ bonds, this only becomes a problem when applying the model to low temperature deposited films that usually contain larger amounts of residual hydrogen than films deposited at high temperature. The a-SiN $\mathrm{X}_{\mathrm{X} 1.33}$ thin films deposited according to Eq. (1) contain negligible amounts of hydrogen due to high temperature deposition and therefore, the model given by Eq. (4) is valid for the proposed fabrication approach. The experiments by Gardeniers, verified Eq. (4) using a value for the refractive index of a-Si equal to that of crystalline silicon or $n_{0}=3.9$ (at $\lambda_{0}=633 \mathrm{~nm}$ ), by measuring the values of $\mathrm{N}: \mathrm{Si}=\mathrm{x}$, in the films they grew, measuring the refractive indices of those films and correlating the measured refractive indices to the calculated ones from Eq. (4) using the measured values of $x$ as input to Eq. (4). Using the results from Gardeniers together with Eqs. (3-4), it becomes possible to calculate the N:Si ratio value $x$, in the silicon rich a$\mathrm{SiN}_{\mathrm{X}<1.33}$, that yields the nearly optimal refractive index as a function of wavelength curve shown in Fig. 10, for achieving refractive index matching with AlN between the sapphire substrate and device silicon.

The N:Si ratio value in the nearly optimal thin film that yields the curve in Fig. 10 is given as $\mathrm{N}: \mathrm{Si}=0.62$, corresponding to a-SiN $\mathrm{S}_{0.62}$. The nearly optimal refractive index as a function of wavelength needed for refractive index matching between sapphire and silicon will be provided by a-SiN 0.62 , however, it is also necessary to consider the extinction coefficient of the antireflective layer now having a reduced optical bandgap compared to stoichiometric a- 


\section{Refractive index of $\mathrm{MgF}_{2}$, sapphire, AIN, $\mathrm{SiN}_{\mathrm{x}=0.62}$ and $\mathrm{Si}$}

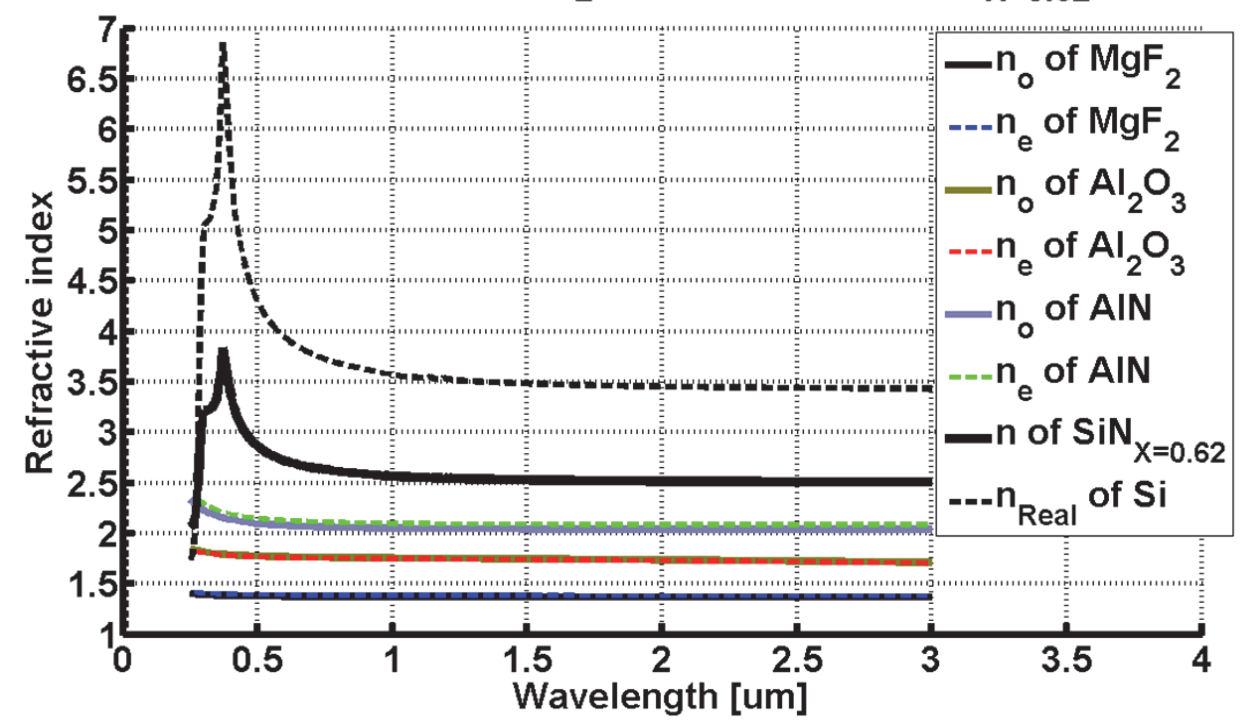

Fig. 10. Real refractive indices for $\mathrm{MgF}_{2}$, sapphire, $\mathrm{AlN}$, a-SiN 0.62 and silicon are shown as a function of the optical wavelength.

$\mathrm{SiN}_{1.33}$, before calculating the back-illuminated optical transmittance of the novel Si-(AlN/a$\mathrm{SiN}_{0.62}$ )-sapphire substrate.

Data for the extinction coefficient as a function of wavelength was not collected in the aSiNX samples deposited by high temperature LPCVD according to Eq. (1) by Gardeniers, however, it is still possible to infer the absorbance of the a-SiN $\mathrm{N}_{0.62}$ antireflective layer from Fig. 10, using data collected by Verlaan, who used HFCVD to deposit a-SiN $\mathrm{N}_{0.62}$ with identical stoichiometry to the nearly optimal antireflective layer in Fig. 10, and measured the extinction coefficient of the sample over the visible wavelength range from 400-650 nm. (Verlaan et al., 2007) Although HFCVD used by Verlaan maintains the substrate at a lower temperature of $230{ }^{\circ} \mathrm{C}$ during deposition compared to high temperature LPCVD used by Gardeniers, the resulting a-SiN $\mathrm{N}_{\mathrm{X}}$ from HFCVD has a density approaching the density of material deposited by high temperature LPCVD while retaining more hydrogen. Despite these differences between LPCVD and HFCVD deposited thin films, the a-SiN 0.62 sample data from Verlaan may be used to infer the expected absorbance as a function of wavelength of the LPCVD deposited a-SiN 0.62 antireflective layer from Fig. 10. To calculate the expected absorbance as a function of wavelength for the a-SiN 0.62 antireflective layer in the Tauc absorption region from $250 \mathrm{~nm}$ to $\lambda_{\mathrm{Eg}}$, from Verlaan's data, the optical bandgap $E_{\mathrm{g}-\mathrm{opt}}$ of the HFCVD deposited a-SiN $\mathrm{N}_{0.62}$ must first be calculated using the Tauc equation given in Eq. (5). (Tauc, 1974)

$$
\sqrt{\hbar \omega \alpha(\omega)}=\sqrt{B}\left(\hbar \omega-E_{g-o p t}\right)
$$


In Eq. (5), $\hbar$ is the reduced Planck constant, $\omega=2 \pi \nu$ is the angular frequency, $E_{\text {g-opt }}$ is the optical bandgap in $\mathrm{eV}$ and $B$ is a slope parameter with units of $\left[\mathrm{cm}^{-1} \mathrm{eV}^{-1}\right]$. Table 2 lists the measured extinction coefficient as a function of the optical wavelength from 400-650 $\mathrm{nm}$ for HFCVD deposited a-SiN 0.62 and substrate temperature of $230^{\circ} \mathrm{C}$.

$\begin{array}{cccc}\text { Wavelength }(\mathrm{nm}) & \text { Extinction coefficient } & \text { Wavelength }(\mathrm{nm}) & \text { Extinction coefficient } \\ 400 & 0.12 & 550 & 0.012 \\ 425 & 0.09 & 575 & 0.007 \\ 450 & 0.064 & 600 & 0.005 \\ 475 & 0.043 & 625 & 0.003 \\ 500 & 0.030 & 650 & 0.002 \\ 525 & 0.018 & & \end{array}$

Table 2. Extinction coefficient of HFCVD deposited a-SiN $\mathrm{S}_{0.62}$ (Verlaan et al., 2007)

Using the measured data for a-SiN 0.62 from Table 2 and knowing that the extinction coefficient of a material is related to its absorption coefficient as $0.5\left(\alpha / k_{0}\right)$, it is possible to plot the left side of Eq. (5) as a function of energy as shown in Fig. 11. Fitting a straight line to the linear part of the scatter plot of points in Fig. 11 and extrapolating to the $x$-axis yields the optical bandgap $E_{\text {g-opt }}=2.1 \mathrm{eV}$ for the HFCVD deposited a-SiN $\mathrm{N}_{0.62}$ from Verlaan. Using the optical bandgap value $E_{\mathrm{g}-\mathrm{opt}}=2.1 \mathrm{eV}$ for a-SiN 0.62 and corresponding wavelength $\lambda_{\mathrm{Eg}}=$ $590 \mathrm{~nm}$, the absorption coefficient as a function of wavelength for the Tauc absorption region is calculated using Eq. (5) and plotted from $250 \mathrm{~nm}$ to $\lambda_{\mathrm{Eg}}=590 \mathrm{~nm}$ in Fig. 12 by fitting to the measured data from Table 2.

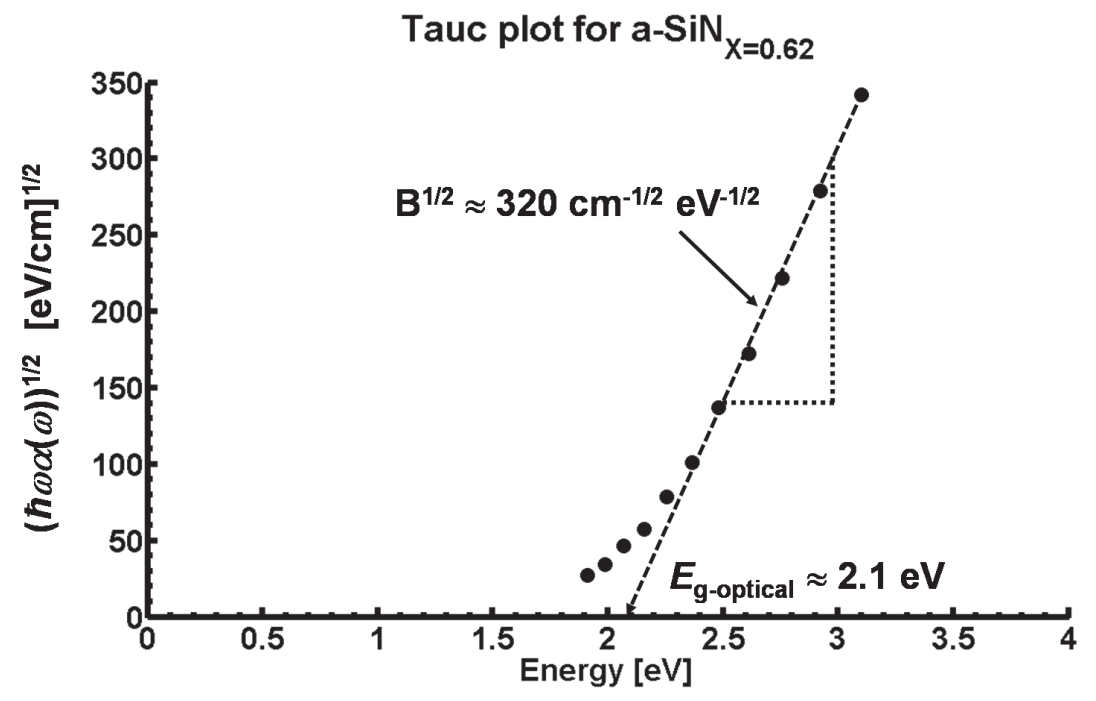

Fig. 11. Determination of the optical bandgap $E_{\text {g-opt }}$ using Tauc plot for a-SiN $\mathrm{N}_{0.62}$ deposited by HFCVD. 


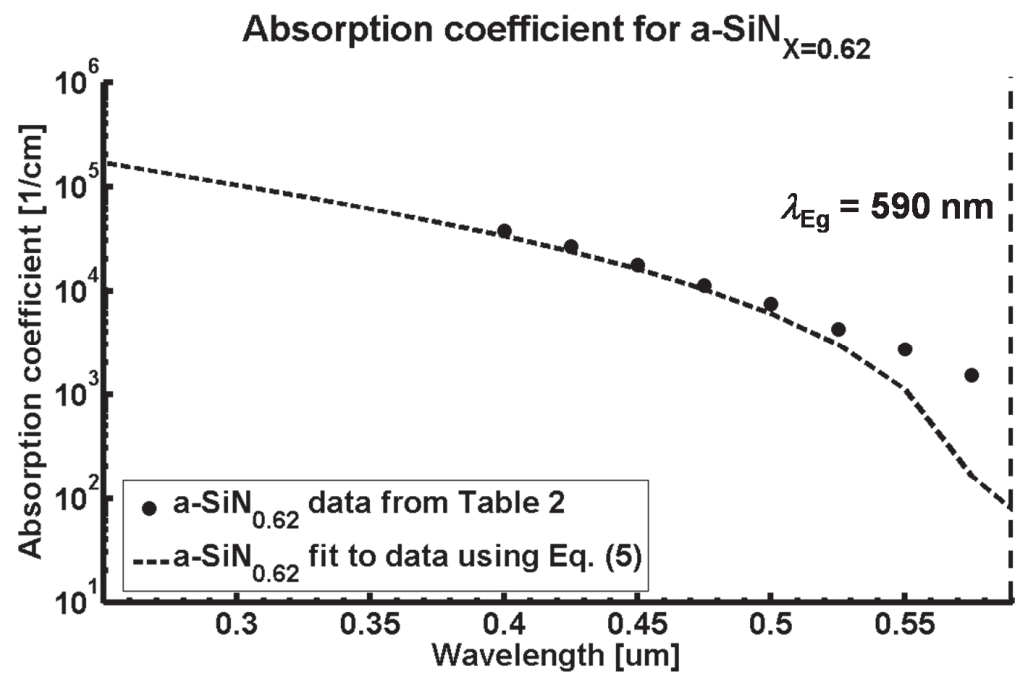

Fig. 12. Tauc absorbance of a-SiN $\mathrm{N}_{0.62}$ calculated using the optical bandgap $E_{\mathrm{g} \text {-opt }}=2.1 \mathrm{eV}$ from Fig. 11.

It will be assumed that the high temperature LPCVD deposited a-SiN $\mathrm{N}_{0.62}$ antireflective layer from Fig. 10 is characterized by a similar absorption coefficient over the Tauc absorption region from $250 \mathrm{~nm}$ to $\lambda_{\mathrm{Eg}}=590 \mathrm{~nm}$ as calculated in Fig. 12 for low temperature HFCVD deposited material, where $\alpha \approx 1 \times 10^{5} \mathrm{~cm}^{-1}$ at $250 \mathrm{~nm}$. In practice, the absorption coefficient in the Tauc absorption region for high temperature LPCVD deposited a-SiN $\mathrm{N}_{0.62}$ should be lower than the calculation in Fig. 12, which represents a worst case scenario. Assuming the worst case of high absorption as calculated in Fig. 12, entails that for an a-SiN $\mathrm{N}_{0.62}$ antireflective layer thickness below $50 \mathrm{~nm}$, the absorbance may still be considered negligible for wavelengths between $250 \mathrm{~nm}$ to $\lambda_{\mathrm{Eg}}=590 \mathrm{~nm}$ and the layer can therefore be modeled as a lossless dielectric. Equation (6) expresses the impedance of a material as a function of the real refractive index $n(\lambda)$, and the absorption coefficient $\alpha(\lambda)$.

$$
\eta(\lambda)=\sqrt{\frac{\mu_{0}}{\varepsilon_{0}\left(n(\lambda)-j \frac{1}{2} \frac{\alpha(\lambda)}{k_{0}}\right)^{2}}}
$$

To calculate the optical power transmittance of TE and TM waves into silicon for the backilluminated $\left(\mathrm{MgF}_{2}\right)$-sapphire-(AlN/a-SiN $\left.\mathrm{Al62}_{2}\right)-\mathrm{Si}$ substrate, the full wave transfer matrix $M_{\text {STACK }}$ for the material layers in the substrate has to be obtained. This result needs to be put into a scattering matrix form that yields the reflection coefficients for the incident waves which in turn allow the reflected and transmitted optical power to be calculated. The wave transfer-scattering matrix theory is described in the text by Saleh \& Teich. (Saleh \& Teich, 2007) The matrix $M_{\text {STACK }}$ for air- $\left(\mathrm{MgF}_{2}\right)$-sapphire-(AlN/a-SiN $\left.\mathrm{S}_{0.62}\right)-\mathrm{Si}$ results from multiplying together nine wave transfer matrices including four for propagation through $\mathrm{MgF}_{2}$, sapphire, $\mathrm{AlN}, \mathrm{SiN}_{\mathrm{X}}$ and five matrices for the material interfaces as shown in Eq. (7). 


$$
M_{\eta-S T A C K}=\left[\begin{array}{ll}
A & B \\
C & D
\end{array}\right]=M_{9} M_{8} M_{7} M_{6} M_{5} M_{4} M_{3} M_{2} M_{1}
$$

The matrices $M_{1}, M_{3}, M_{5}, M_{7}$ and $M_{9}$ represent wave transfer matrices at the air- $\mathrm{MgF}_{2}, \mathrm{MgF}_{2}-$ sapphire, sapphire-AlN, AlN-a-SiN 0.62 and a-SiN 0.62 -silicon interfaces while matrices $M_{2}, M_{4}$, $M_{6}$ and $M_{8}$ are propagation matrices through $\mathrm{MgF}_{2}$, sapphire, AlN and a-SiN 0.62 . All nine matrices are expressed in terms of the complex impedances of the materials given by Eq. (6). Using a Monte Carlo integration approach, it is possible to calculate the back-illuminated optical transmittance into the APD device silicon as a function of wavelength, for TE waves normally incident to the sapphire substrate plane of the mesa APD pixel from Fig. 7, as shown in Fig. 13.

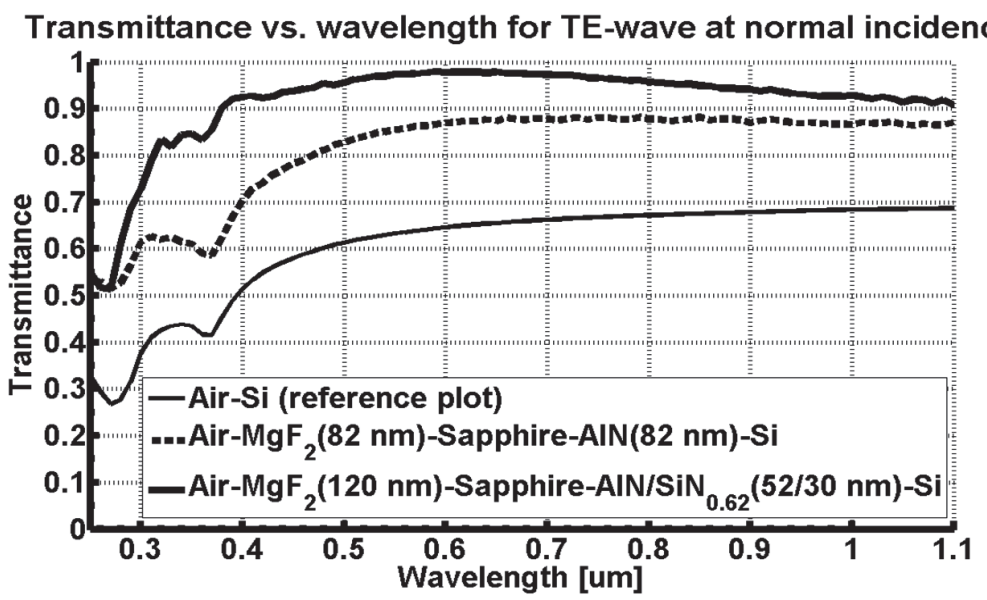

Fig. 13. Optical power transmittance into silicon of a TE wave normally incident from air to the back-illuminated APD substrate.

From the calculation in Fig. 13, it is evident that using silicon rich a- $\mathrm{SiN}_{0.62}$ prepared by high temperature LPCVD as an antireflective layer together with AIN, provides the required refractive index matching to enable very high transmittance, back-illuminated silicon-on-sapphire wafer substrates, while retaining a sufficiently high optical bandgap to be treated as a lossless dielectric in this application, as calculated in Figs. 11-12. The silicon-on-sapphire substrate represented by the thick solid curve in Fig. 13, having an AlN/a-SiN 0.62 antireflective bilayer of $52 / 30 \mathrm{~nm}$ thickness respectively between sapphire and silicon and $120 \mathrm{~nm}$ thick $\mathrm{MgF}_{2}$ antireflective layer between air and sapphire, provides significantly improved back-illuminated transmittance into silicon as compared to the silicon-on-sapphire substrate represented by the dashed curved in Fig. 13, having only an $82 \mathrm{~nm}$ thick $\lambda / 4$-AlN antireflective layer between sapphire and silicon and an $82 \mathrm{~nm}$ thick $\lambda / 4-\mathrm{MgF}_{2}$ antireflective layer between air and sapphire. Other advantages of using silicon rich a-SiN $\mathrm{X}_{\mathrm{X}<1.33}$ films include lower tensile strain than stoichiometric films which is important for reducing the injection of vacancy and interstitial defects in silicon. High

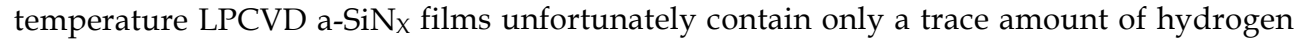
compared to PECVD and HFCVD films, and hydrogen is very useful for bulk and surface 
passivation of silicon defects and the lengthening of carrier lifetimes in silicon. It is particularly challenging to optimize a-SiN $\mathrm{N}_{X}$ films for high transmittance antireflective layers, that are better prepared using high temperature LPCVD and also for bulk and surface silicon passivation with hydrogen, which require low temperature deposition using PECVD or HFCVD. Such novel substrates however, represent an enabling technology for the next generation of high performance Si/SiGe APD focal plane array imagers.

\section{Optical crosstalk in silicon-on-sapphire APD-FPAs}

The phenomenon of optical crosstalk in high resolution avalanche photodiode arrays is well known and has been the subject of extensive theoretical and experimental study. (Akil et al., 1998, 1999; Lahbabi et al., 2000; Rech et al., 2008) Optical crosstalk in APD arrays results primarily from photon emission that occurs during impact ionization in the avalanche carrier multiplication process, erroneously triggering neighboring APD pixels and thereby producing noise. The increased APD-FPA noise from optical crosstalk might prevent high sensitivity imaging. Past scientific literature has mainly addressed the phenomenon of direct pixel-to-pixel optical crosstalk which occurs between immediately adjacent detector pixels by line of sight light propagation, however, indirect optical crosstalk might also be present. Indirect optical crosstalk is more difficult to block and occurs from light undergoing multiple reflections in the planar sapphire substrate waveguide. Two forms of indirect optical crosstalk exist in the silicon-on-sapphire APD-FPA due to multiple reflections and they include reflected light generated in the multiplication region of the APD during a detection event as well as reflected incident ambient illumination as shown in Figs. $14-15$ respectively.

The back-illuminated silicon-on-sapphire material system readily enables the design of $\mathrm{Si} / \mathrm{SiGe} \mathrm{APD}$ focal plane arrays with zero direct pixel-to-pixel optical crosstalk by line of sight propagation as understood from Fig. 14, where the $\mathrm{Al}$ or $\mathrm{Cu}$ metal anode grid across the mesa APD detector array performs the important secondary function of blocking direct

(NOT TO SCALE)

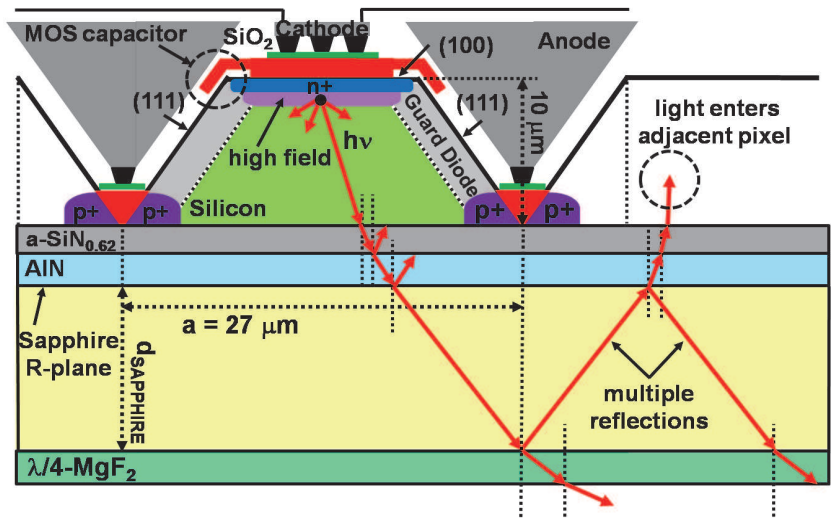

Fig. 14. Indirect optical crosstalk from APD emitted light coupled into the sapphire substrate waveguide. 


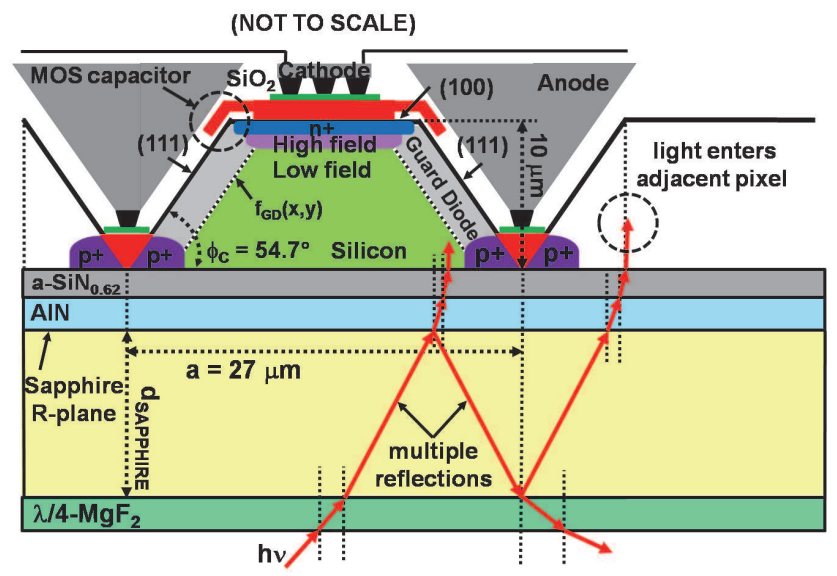

Fig. 15. Indirect optical crosstalk from ambient incident illumination coupled into the sapphire substrate waveguide.

line of sight light propagation between adjacent detectors. In Fig. 14, the mesa APD is shown by approximation to emit light isotropically from a central point in the high electric field avalanche multiplication region. A fraction of the emitted photons are coupled into the planar sapphire substrate waveguide where they might undergo multiple reflections and subsequent transmission into the neighboring silicon mesa APD pixels. In Sec. 3.1-3.2, the fraction of APD emitted light during linear or Geiger-mode detection events transmitted into neighboring 27 $\mu \mathrm{m}$ mesa APD pixels is calculated for planar Si-(AlN)-sapphire and Si-(AlN/a-SiN $\mathrm{N}_{0.62}$ )sapphire substrates with back-side $\lambda / 4-\mathrm{MgF}_{2}$ antireflective layer. In Sec. 4 , the fraction of incident light on a $27 \mu \mathrm{m}$ mesa APD from an isotropic point source at infinity, transmitted into neighboring detectors as shown in Fig. 15, is calculated for planar Si-(AlN)-sapphire and Si(AlN/SiN 0.62 )-sapphire substrates with back-side $\lambda / 4-\mathrm{MgF}_{2}$ antireflective layer.

Indirect optical crosstalk through the planar sapphire substrate can be reduced by thinning the sapphire substrate and fabricating microlenses beneath each APD detector pixel as shown in Fig. 7. Although microlenses increase APD-FPA performance as described in Sec. 2.1, they also increase the cost and fabrication complexity of the detector arrays. (Stern \& Cole, 2008) It will be shown in detailed analysis and calculations in Sec. 5, that indirect optical crosstalk through the planar sapphire substrate waveguide from light generated by impact ionization or from ambient illumination will not increase detector noise to levels that prevent high sensitivity imaging and therefore, fabricating sapphire microlenses is not an imperative for achieving low noise, high resolution Si/SiGe APD-FPAs using backilluminated silicon-on-sapphire.

\subsection{Indirect optical crosstalk from APD emitted light, coupled into the sapphire waveguide; Si-(AIN)-sapphire}

The fraction of light emitted by an APD during detection events, coupled into the sapphire substrate waveguide and transmitted to neighboring detectors thereby contributing to indirect optical crosstalk, can be calculated by modeling and simulation of the mesa APD detector pixel. Using the Monte Carlo method, it is possible to calculate how photons are emitted from 
the APD multiplication region and transmitted into adjacent mesa APD detector pixels as shown in Fig. 16, for Si-(AIN)-sapphire substrates with back-side $\lambda / 4-\mathrm{MgF}_{2}$ antireflective layer. The thickness for both the AlN and $\mathrm{MgF}_{2}$ antireflective layers is taken to be $82 \mathrm{~nm}$ as indicated in Fig. 16. The high electric field mesa APD multiplication region shown in Fig. 16 is 300-500 $\mathrm{nm}$ thick and consists of more highly doped p-type silicon formed by boron impurity diffusion. (Stern \& Cole, 2008) Although impact ionization resulting in avalanche multiplication of charge carriers as well as photon emission, occurs throughout the volume of the high electric field region, for modeling purposes, it will be assumed that photons are only emitted isotropically from a single point in the center of the multiplication region located at a height $h_{\mathrm{M}}=9 \mu \mathrm{m}$, above the silicon pixel base plane as shown in Fig. 16 .

Using the Monte Carlo simulation approach, points are randomly generated in the silicon base plane area of the $27 \mu \mathrm{m}$ mesa APD. Trajectories of light propagation or optical kvectors are created by connecting straight lines from the isotropic point source in the multiplication region to the randomly generated points in the silicon base plane area of the $27 \mu \mathrm{m}$ mesa APD as shown in Fig. 17.

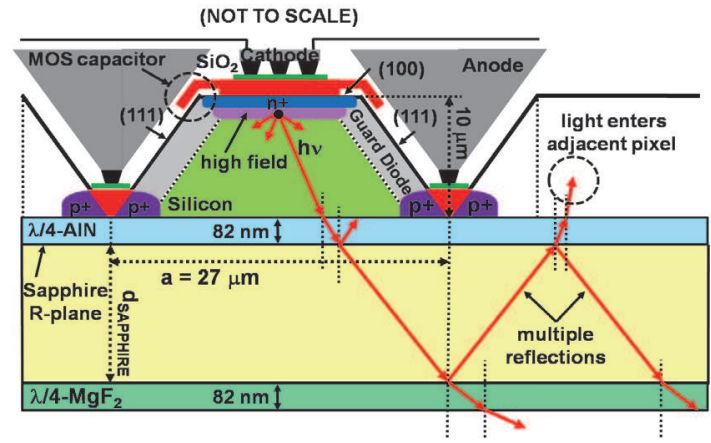

Fig. 16. Indirect optical crosstalk from APD emitted light coupled into the (AIN)-sapphire substrate waveguide.

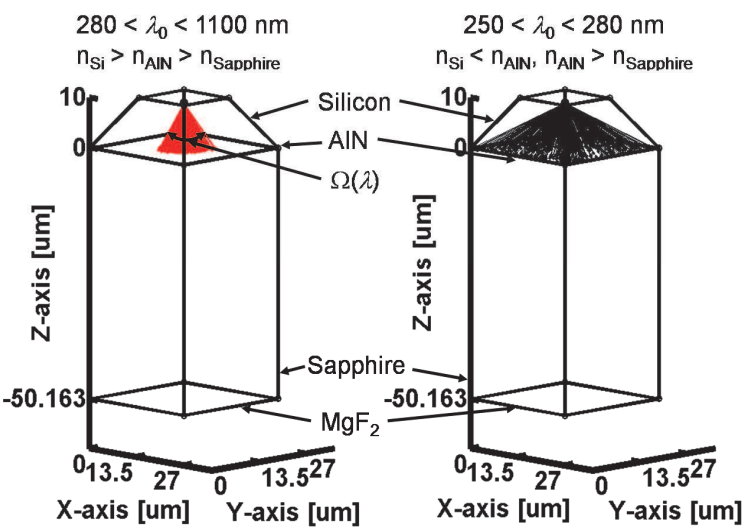

Fig. 17. 3-D ray tracing shows the optical k-vector transmission cone for $280<\lambda_{0}<1100 \mathrm{~nm}$ light into the sapphire substrate. 
In Fig. 17, 3-D ray tracing is used to calculate paths of light propagation for the randomly generated optical k-vectors over a $250<\lambda_{0}<1100 \mathrm{~nm}$ wavelength range, emitted from the APD multiplication region, transmitted into the sapphire substrate and undergoing multiple reflections. The calculation in Fig. 17 shows that light between the wavelengths of 280-1100 nm (left pixel in Fig. 17) emitted from the isotropic point source in the multiplication region, can only exit the mesa APD through the sapphire substrate waveguide if the optical k-vector from the point source is emitted into a cone characterized by a wavelength dependent solid angle $\Omega(\lambda)$, subtended by a corresponding circular base area and having height $h_{\mathrm{M}}=9 \mu \mathrm{m}$ of the isotropic point source. The calculation also shows that for $250<\lambda_{0}<280 \mathrm{~nm}$ wavelengths (right pixel in Fig. 17) light can be transmitted into the sapphire substrate through the major part of the $27 \mu \mathrm{m}$ mesa APD base area because the refractive index of $\mathrm{Si}$ is smaller than AlN as shown in Fig. 10, and the incidence angle at the AlN-sapphire interface does not exceed the critical angle $\theta_{C}$ except when optical k-vectors with $270<\lambda_{0}<280 \mathrm{~nm}$, are incident near the corners of the mesa APD base area. For $280<\lambda_{0}<1100 \mathrm{~nm}$ wavelengths, the refractive index of silicon is larger than AIN as well as sapphire and total internal reflection (TIR) occurs if the incidence angle of the optical k-vector at the silicon-AIN or AlN-sapphire interface exceeds the critical angle $\theta_{C}$. The critical angles $\theta_{C}$ for light emission out of the silicon mesa into the AlN and from AlN into the sapphire substrate, depend on the refractive indices of the silicon, AlN and sapphire material layers, that in turn depend on the optical wavelength as shown in Eq. (8).

$$
\theta_{C}=\operatorname{asin}\left(\frac{n_{2}(\lambda)}{n_{1}(\lambda)}\right) ; \quad n_{1}>n_{2}
$$

In Eq. (8), the light propagates from a material with refractive index $n_{1}$ to a material with refractive index $n_{2}$ where $n_{1}>n_{2}$. Using Eq. (8), the critical angle $\theta_{C}(\lambda)$ is calculated in Fig. 18 as a function of wavelength at the Si-AlN (dashed line) and AlN-sapphire (thin solid line) material interfaces. The effective critical angle $\theta_{C \text {-eff }}(\lambda)$, (thick solid line) shown in Fig. 18, for light transmission from $\mathrm{Si}$ into sapphire through AlN, is equal to the critical angle for light transmission directly from Si into sapphire. The corresponding effective, fractional solid angle $\Omega_{\mathrm{F}}$ for light transmission from Si into sapphire through AlN, as a function of wavelength is calculated as the effective light transmission cone solid angle $\Omega_{\text {eff }}=\pi\left(\theta_{\text {-eff }}(\lambda)\right)^{2}$ divided by $4 \pi \mathrm{sr}$ solid angle of the sphere into which the isotropic point source emits, as shown in Fig. 19.

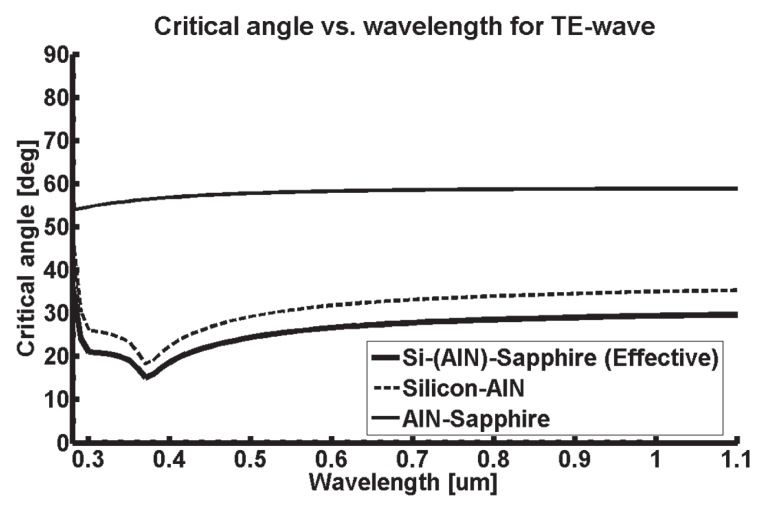

Fig. 18. Critical angle for Si-AlN, AlN-Sapphire, Si-Sapphire. 


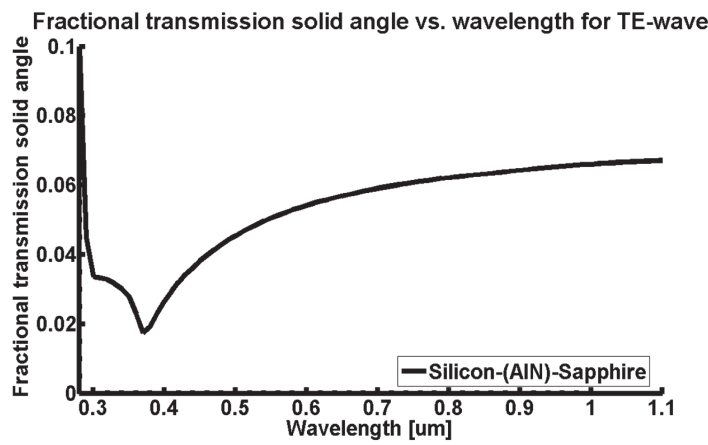

Fig. 19. Fractional solid angle of the light transmission cone.

Reducing the fractional solid angle of the light transmission cone calculated in Fig. 19, would help to prevent optical k-vectors with large incidence angle at the Si-AlN and AlN-sapphire interfaces from propagating into the sapphire substrate where they can undergo multiple reflections and transmission into distant APD detectors in the array to produce optical crosstalk at a distance. Reducing the effective fractional solid angle of the light transmission cone requires a large refractive index contrast ratio between the $\mathrm{Si}$ semiconductor device layer and the optically transparent supporting substrate and does not depend on the thin antireflective layers such as AIN between the $S i$ and sapphire where $n_{\mathrm{Si}}>n_{\mathrm{AIN}}>n_{\mathrm{SAPPHIRE}}$.

It will be assumed that any optical k-vectors reflected back into the silicon APD by TIR will not have a second pass, or opportunity to escape the mesa pixel by transmission into the sapphire substrate waveguide and even if such TIR optical k-vectors might be transmitted through the (111) sidewalls of the mesa, the light will subsequently be blocked by the anode metal grid and will not contribute to optical crosstalk. Thus, only the optical k-vectors emanating from the isotropic point source in the APD multiplication region and contained by the light transmission cone calculated in Fig. 19 for $280<\lambda_{0}<1100 \mathrm{~nm}$ wavelengths or contained by the solid angle subtended by most of the silicon mesa base area for $250<\lambda_{0}<280 \mathrm{~nm}$ wavelengths, will couple into the sapphire substrate and therefore contribute to the indirect optical crosstalk. Using the result from Fig. 19, it is possible to calculate the fraction of light emitted by the isotropic point source in the mesa APD multiplication region that is transmitted through the sapphire substrate to other APD detectors in the array as a function of wavelength. Multiple reflections may occur in the sapphire substrate for the APD emitted light, and such reflections might not necessarily be bounded by the areas of the eight numbered and immediately adjacent $27 \mu \mathrm{m}$ mesa APD detector pixels shown in Fig. 20.

$3 \times 3$ silicon APD detector array showing mesa outlines

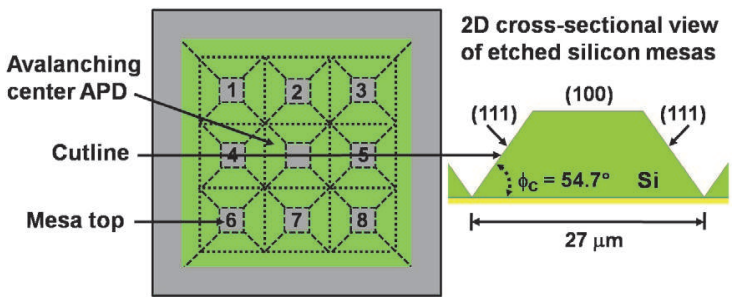

Fig. 20. 3x3 array showing eight immediately adjacent APDs. 


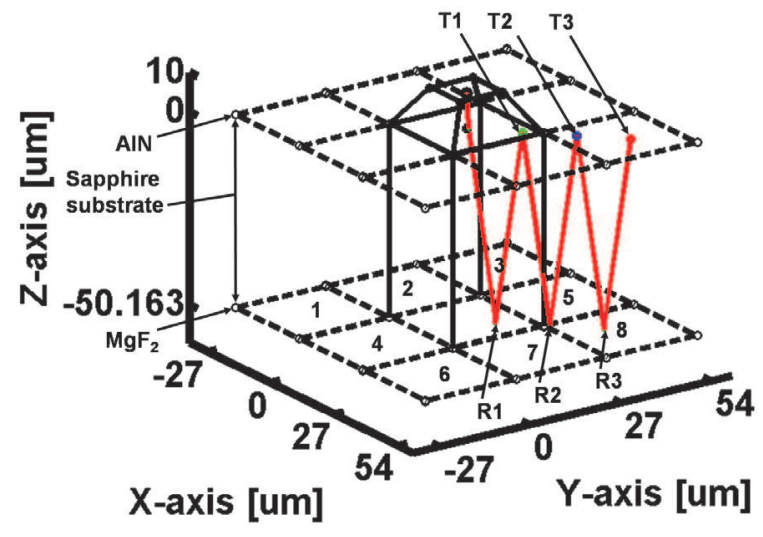

Fig. 21. 3-D ray tracing shows simulated multiple reflections.

The optical transmittance into adjacent detectors numbered 1-8 as well as other detectors outside of the immediately adjacent numbered pixels shown in Fig. 20, is obtained by calculating the fraction of light transmitted into silicon after each successive reflection cycle in the sapphire substrate for an optical k-vector as shown in Fig. 21. The first reflection cycle in the sapphire substrate is indexed as $T_{1}$ followed by the second and third cycles with index $T_{2}, T_{3} \ldots T_{\mathrm{N}}$ where $T_{\mathrm{N}}$ is the highest calculated reflection in the substrate. The results from Fig. 19 and Fig. 21, are used to calculate the fraction of light emitted by the isotropic point source in the mesa APD multiplication region, that will be transmitted through the sapphire substrate to other APD detectors in the array as a function of wavelength as shown in Figs. 22-23.

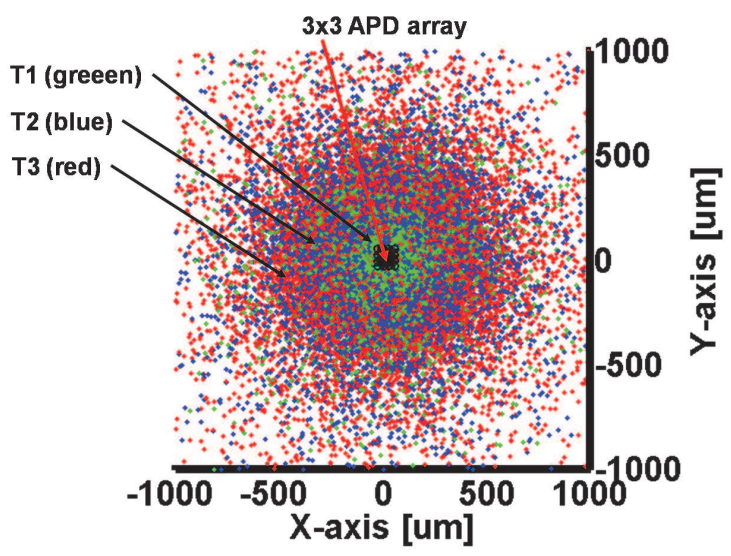

Fig. 22. Average crosstalk distance for $50 \mu \mathrm{m}$ thick sapphire. 


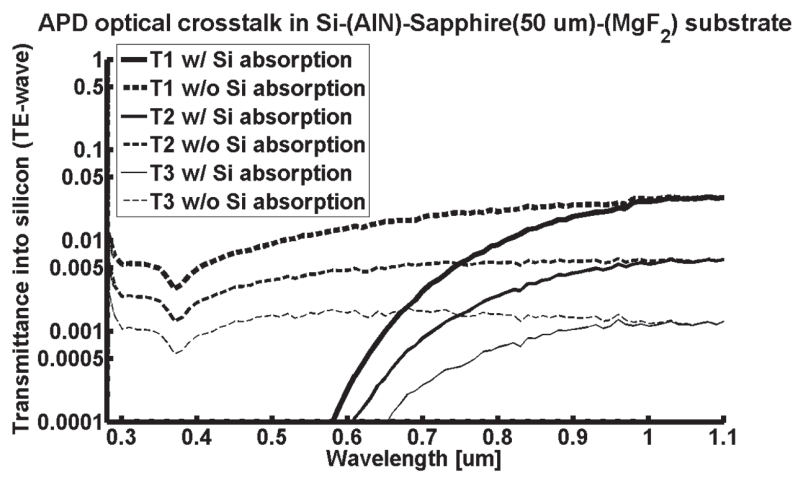

Fig. 23. Indirect APD optical crosstalk in $50 \mu \mathrm{m}$ thick sapphire.

The average distance of light transmittance points $T 1, T 2$ and $T 3$ into the neighboring APD pixels, from the avalanching center mesa APD (shown in Fig. 20) is calculated in Fig. 22 for a $50 \mu \mathrm{m}$ thick sapphire substrate. In Fig. 23, the fraction of light emitted by the isotropic point source in the mesa APD multiplication region and transmitted to neighboring APD pixels is calculated for a maximum of three reflection cycles, T1, T2 and T3, with and without light absorption in the silicon. (Lahbabi et al., 2000) On the first reflection cycle represented by T1 (shown in Figs. 21-23), between 1-5\% of the isotropically emitted light from the APD multiplication region having wavelength $280-1100 \mathrm{~nm}$, is transmitted into neighboring pixels while the second reflection cycle $T 2$, transmits $0.1-0.5 \%$ and the third reflection cycle T3, transmits $0.05-0.1 \%$ of emitted light into the neighboring pixels. The results in Fig. 24 show that the average distance of $T 1$ for a $10 \mu \mathrm{m}$ thick sapphire substrate corresponds to a radius of a circle contained by the eight adjacent pixels of the avalanching center APD shown in Fig. 20.

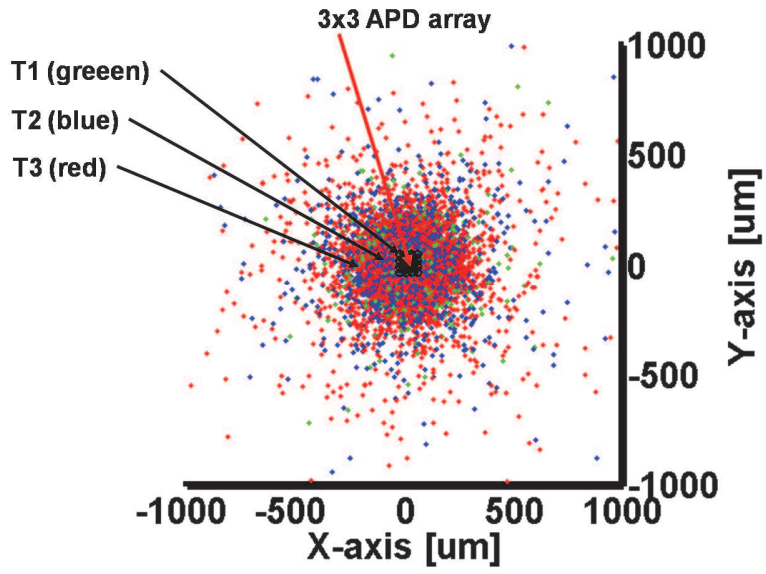

Fig. 24. Average crosstalk distance for $10 \mu \mathrm{m}$ thick sapphire. 


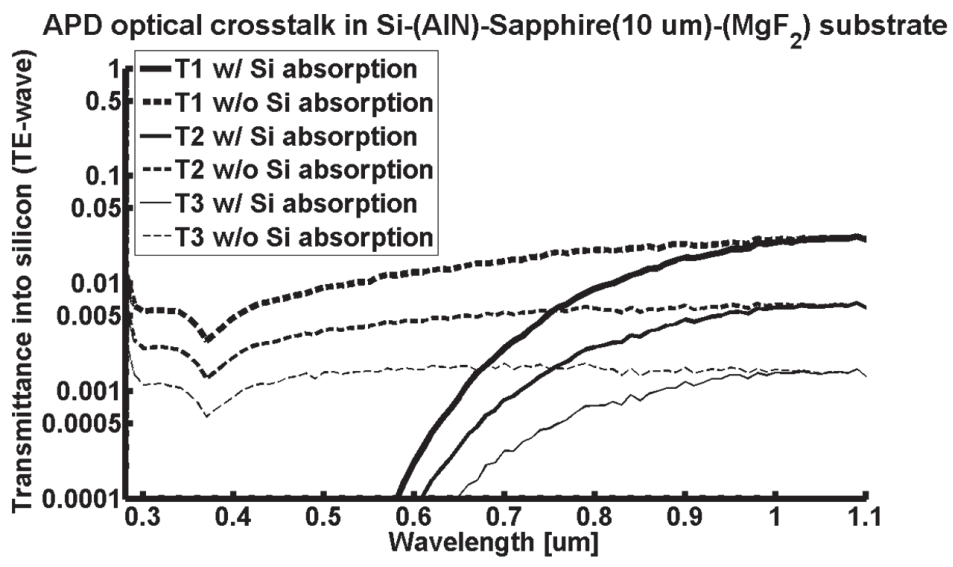

Fig. 25. Indirect APD optical crosstalk in $10 \mu \mathrm{m}$ thick sapphire.

The results in Figs. 22-25 will be analyzed in Sec. 5 for their effect on the signal-to-noise ratio of APD detectors in an array.

\subsection{Indirect optical crosstalk from APD emitted light, coupled into the sapphire waveguide; $\mathrm{Si}$-(AIN/SiN 0.62$)$-sapphire}

The fraction of light emitted by a $27 \mu \mathrm{m}$ mesa APD during detection events, coupled into the sapphire substrate waveguide and transmitted to neighboring detectors thereby contributing to indirect optical crosstalk, can be calculated for very high transmittance Si(AlN/a-SiN ${ }_{0.62}$ )-sapphire substrates with back-side $\lambda / 4-\mathrm{MgF}_{2}$ antireflective layer described in Sec. 2.2, by following an approach similar to that in Sec. 3.1 for Si-(AlN)-sapphire substrates. Using the Monte Carlo method, it is possible to calculate how photons are emitted from the APD multiplication region and transmitted into adjacent mesa APD detector pixels as shown in Fig. 26, for silicon-(AlN/a-SiN $\left.\mathrm{N}_{0.62}\right)$-sapphire substrates with back-side $\lambda / 4-\mathrm{MgF}_{2}$ antireflective layer. The thickness of the $\mathrm{AlN}$, a-SiN 0.62 and $\mathrm{MgF}_{2}$ layers is $d_{\mathrm{AIN}}=52 \mathrm{~nm}, d_{\mathrm{a}-\mathrm{SiN} \_0.62}=30 \mathrm{~nm}$ and $d_{\mathrm{MgF} 2}=120 \mathrm{~nm}$ as indicated in Fig. 26. The high electric field mesa APD multiplication region shown in Fig. 26 is 300-500 nm thick and consists of more highly doped p-type silicon formed by boron impurity diffusion. (Stern \& Cole, 2008) Although impact ionization resulting in avalanche multiplication of charge carriers as well as photon emission, occurs throughout the volume of the high electric field region, for modeling purposes it will be assumed that photons are only emitted isotropically from a single point in the center of the multiplication region located at a height $h_{\mathrm{M}}=9 \mu \mathrm{m}$, above the silicon pixel base plane as shown in Fig. 26.

Using the Monte Carlo simulation approach, points are randomly generated in the silicon base plane area of the $27 \mu \mathrm{m}$ mesa APD. Trajectories of light propagation or optical kvectors are created by connecting straight lines from the isotropic point source in the multiplication region to the randomly generated points in the silicon base plane area of the $27 \mu \mathrm{m}$ mesa APD as shown in Fig. 27. 


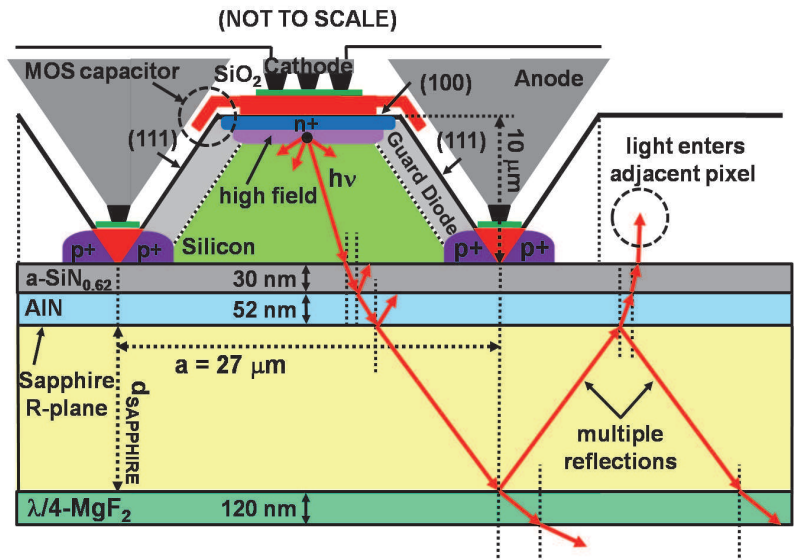

Fig. 26. Indirect optical crosstalk from APD emitted light coupled into the (AlN/a-SiN $\mathrm{N}_{0.62}$ sapphire substrate waveguide.

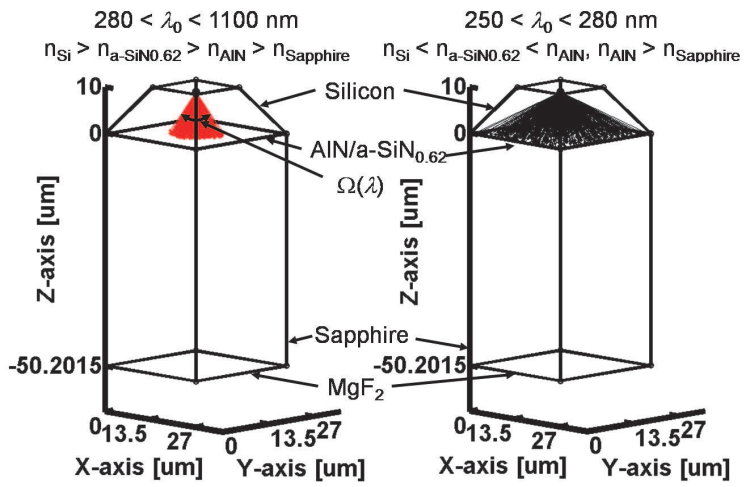

Fig. 27. 3-D ray tracing shows the optical k-vector transmission cone for $280<\lambda_{0}<1100 \mathrm{~nm}$ light into the sapphire substrate.

As in Sec. 3.1, 3-D ray tracing is used in Fig. 27 to calculate paths of light propagation for the randomly generated optical k-vectors over a $250<\lambda_{0}<1100 \mathrm{~nm}$ wavelength range, emitted from the APD multiplication region, transmitted into the sapphire substrate and undergoing multiple reflections. The calculation in Fig. 27 shows that light between the wavelengths of 280-1100 nm (left pixel in Fig. 27) emitted from the isotropic point source in the multiplication region, can only exit the mesa APD through the sapphire substrate waveguide if the optical k-vector from the point source is emitted into a cone characterized by a wavelength dependent solid angle $\Omega(\lambda)$, subtended by a corresponding circular base area and having height $h_{\mathrm{M}}=9 \mu \mathrm{m}$ of the isotropic point source. The calculation also shows that for $250<\lambda_{0}<280 \mathrm{~nm}$ wavelengths (right pixel in Fig. 27) light can be transmitted into the sapphire substrate through the major part of the $27 \mu \mathrm{m}$ mesa APD base area because the refractive index of $\mathrm{Si}$ is smaller than a-SiN 0.62 and $\mathrm{AlN}$ as shown in Fig. 10, and the incidence angle at the AlN-sapphire interface does not exceed the critical angle $\theta_{c}$ except when optical 
k-vectors with $270<\lambda_{0}<280 \mathrm{~nm}$, are incident near the corners of the mesa APD base area. For $280<\lambda_{0}<1100 \mathrm{~nm}$ wavelengths, the refractive index of silicon is larger than a-SiN $\mathrm{N}_{0.62}$, AIN as well as sapphire and total internal reflection (TIR) occurs if the incidence angle of the optical k-vector at the silicon-a-SiN $\mathrm{N}_{0.62}$, silicon-AlN or AlN-sapphire interface exceeds the critical angle $\theta_{C}$. The critical angles $\theta_{C}$ for light emission out of the silicon mesa into the a$\mathrm{SiN}_{0.62}$, from a-SiN $\mathrm{S}_{0.62}$ into AlN and from AlN into the sapphire substrate, depend on the refractive indices of the silicon, a-SiN $\mathrm{S}_{0.62}$, AlN and sapphire material layers, that in turn depend on the optical wavelength as given by Eq. (8). In Eq. (8) the light propagates from a material with refractive index $n_{1}$ to a material with refractive index $n_{2}$ with $n_{1}>n_{2}$.

Using Eq. (8), the critical angle $\theta_{C}(\lambda)$ is calculated in Fig. 28 as a function of wavelength at the different material interfaces. The effective critical angle $\theta_{\text {C-eff }}(\lambda)$, (thick solid line) shown in Fig. 28, for light transmission from $\mathrm{Si}$ into sapphire through a-SiN $\mathrm{N}_{0.62}$ and $\mathrm{AlN}$, is equal to the critical angle for light transmission directly from $\mathrm{Si}$ into sapphire. The corresponding effective, fractional solid angle $\Omega_{\mathrm{F}}$ for light transmission from Si into sapphire through AIN, as a function of wavelength is calculated as the effective light transmission cone solid angle $\Omega_{\text {eff }}=\pi\left(\theta_{\text {-eff }}(\lambda)\right)^{2}$ divided by $4 \pi$ sr solid angle of the sphere into which the isotropic point source emits, as shown in Fig. 29.

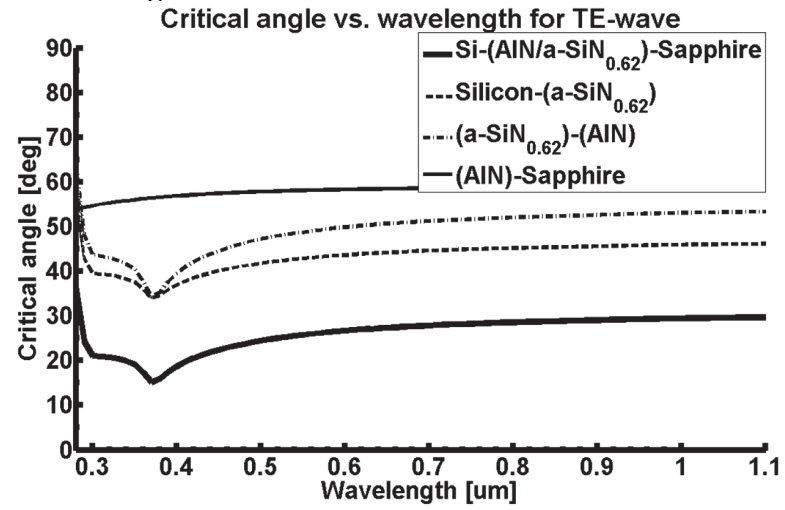

Fig. 28. Critical angles for material interfaces in the substrate.

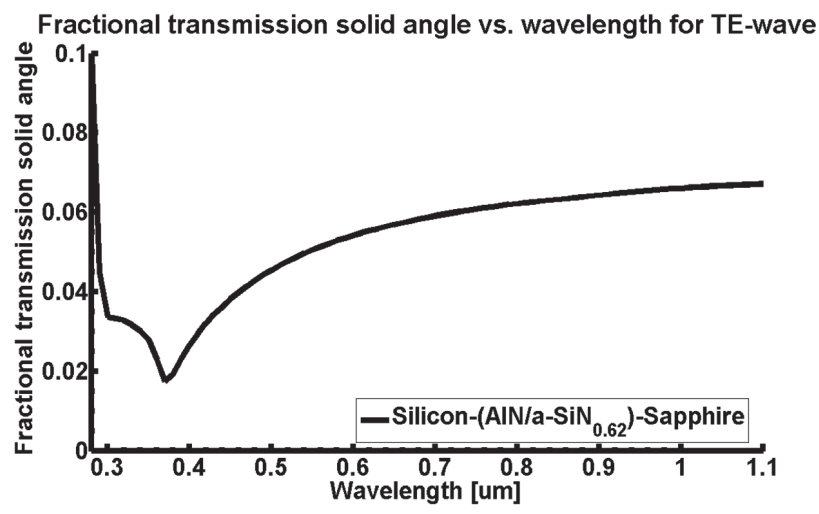

Fig. 29. Fractional solid angle of the light transmission cone. 
Reducing the fractional solid angle of the light transmission cone calculated in Fig. 29, would help to prevent optical k-vectors with large incidence angle at the silicon-a-SiN $\mathrm{S}_{0.62}$, a$\mathrm{SiN}_{0.62}$-AIN and AlN-sapphire interfaces from propagating into the sapphire substrate where they can undergo multiple reflections and transmission into distant APD detectors in the array to produce optical crosstalk at a distance. Reducing the effective fractional solid angle of the light transmission cone requires a large refractive index contrast ratio between the Si semiconductor device layer and the optically transparent supporting substrate and does not depend on the thin antireflective layers such as a-SiN $\mathrm{N}_{0.62}$ and AlN between the Si and sapphire where $n_{\mathrm{Si}}>n_{\mathrm{a}-\mathrm{SiN} \_0.62}>n_{\mathrm{AIN}}>n_{\mathrm{SAPPHIRE}}$.

It will be assumed as in Sec 3.1 that any optical k-vectors reflected back into the silicon APD by TIR will not have a second pass, or opportunity to escape the mesa pixel by transmission into the sapphire substrate waveguide and even if such TIR optical k-vectors might be transmitted through the (111) sidewalls of the mesa, the light will subsequently be blocked by the anode metal grid and will not contribute to optical crosstalk. Thus, only the optical kvectors emanating from the isotropic point source in the APD multiplication region and contained by the light transmission cone calculated in Fig. 29 for $280<\lambda_{0}<1100 \mathrm{~nm}$ wavelengths or contained by the solid angle subtended by most of the silicon mesa base area for $250<\lambda_{0}<280 \mathrm{~nm}$ wavelengths, will couple into the sapphire substrate and therefore contribute to the indirect optical crosstalk. Using the result from Fig. 29, it is possible to calculate the fraction of light emitted by the isotropic point source in the mesa APD multiplication region that is transmitted through the sapphire substrate to other APD detectors in the array as a function of wavelength. Multiple reflections may occur in the sapphire substrate for the APD emitted light, and such reflections might not necessarily be bounded by the areas of the eight numbered and immediately adjacent $27 \mu \mathrm{m}$ mesa APD detector pixels shown in Fig. 30.

\section{$3 \times 3$ silicon APD detector array showing mesa outlines}

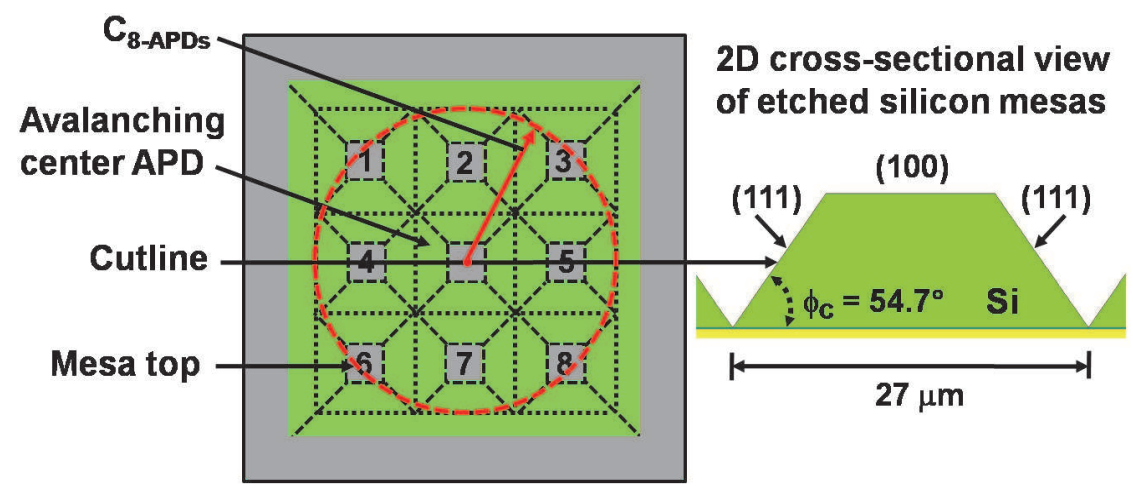

Fig. 30. 3x3 array showing eight immediately adjacent APDs. 


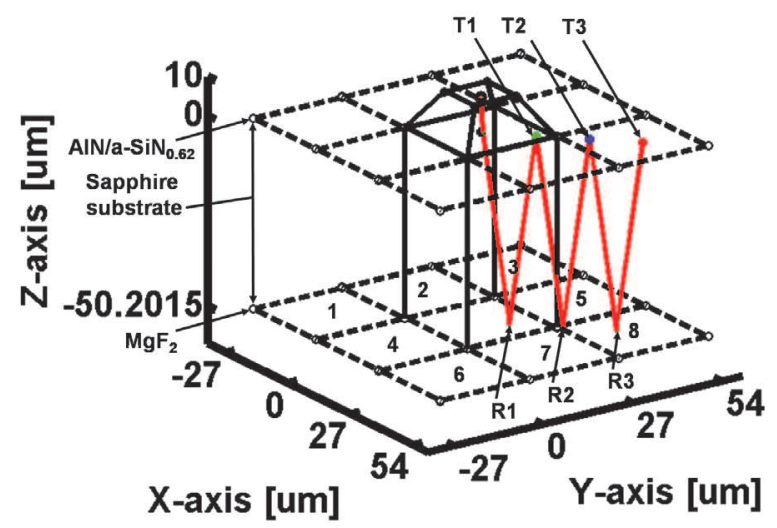

Fig. 31. 3-D ray tracing shows simulated multiple reflections.

The optical transmittance into adjacent detectors numbered 1-8 as well as other detectors outside of the immediately adjacent numbered pixels shown in Fig. 30, is obtained by calculating the fraction of light transmitted into silicon after each successive reflection cycle in the sapphire substrate for an optical k-vector as shown in Fig. 31, using the wave transfer matrix-scattering matrix method discussed in Sec. 2.2. The first reflection cycle in the sapphire substrate is indexed as $T_{1}$ followed by the second and third cycles with index $T_{2}, T_{3} \ldots T_{\mathrm{N}}$ where $T_{\mathrm{N}}$ is the highest calculated reflection in the substrate. The results from Fig. 29 and Fig. 31, are used to calculate the fraction of light emitted by the isotropic point source in the mesa APD multiplication region, that will be transmitted through the sapphire substrate to other APD detectors in the array as a function of wavelength as shown in Figs. 32-33.

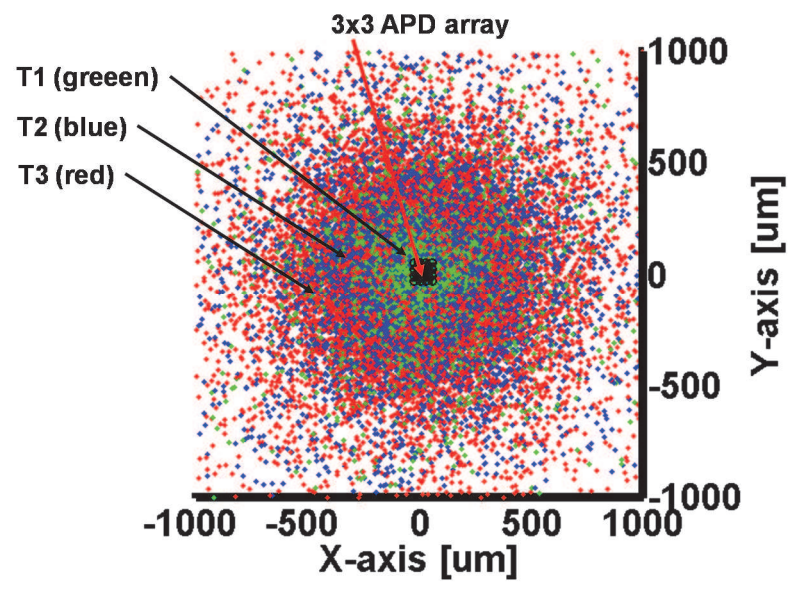

Fig. 32. Average crosstalk distance for $50 \mu \mathrm{m}$ thick sapphire. 


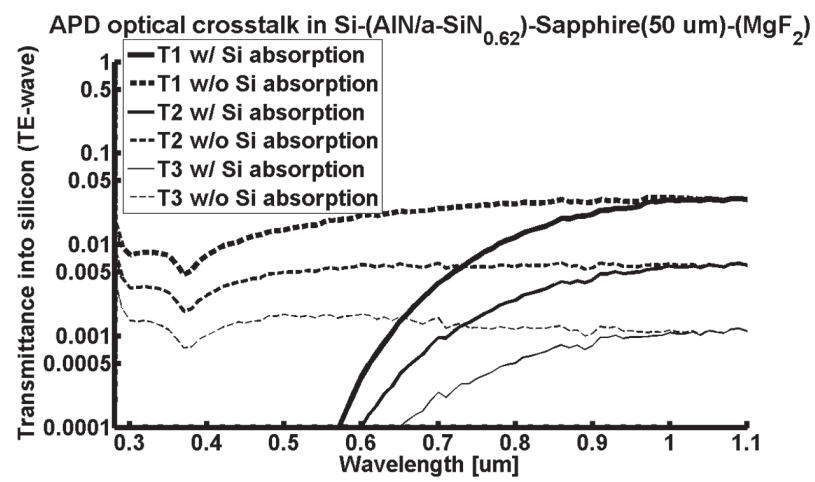

Fig. 33. Indirect APD optical crosstalk in $50 \mu \mathrm{m}$ thick sapphire.

The average distance of light transmittance points $T 1, T 2$ and $T 3$ into the neighboring APD pixels, from the avalanching center mesa APD (shown in Fig. 30) is calculated in Fig. 32 for a $50 \mu \mathrm{m}$ thick sapphire substrate. In Fig. 33, the fraction of light emitted by the isotropic point source in the mesa APD multiplication region and transmitted to neighboring APD pixels is calculated for a maximum of three reflection cycles, T1, T2 and T3, with and without light self-absorption in the silicon. (Lahbabi et al., 2000) On the first reflection cycle represented by $T 1$ (shown in Figs. 31-33), between 1-5\% of the isotropically emitted light from the APD multiplication region having wavelength $280-1100 \mathrm{~nm}$, is transmitted into neighboring pixels while the second reflection cycle $T 2$, transmits $0.1-0.5 \%$ and the third reflection cycle T3, transmits $0.05-0.1 \%$ of emitted light into the neighboring pixels. The results in Fig. 34 show that the average distance of $T 1$ for a $10 \mu \mathrm{m}$ thick sapphire substrate corresponds to a radius of a circle contained by the eight adjacent pixels of the avalanching center APD shown in Fig. 30.

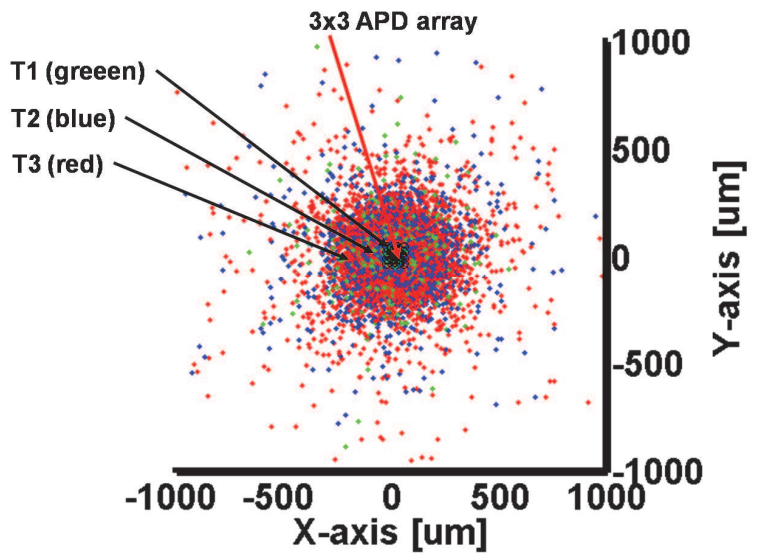

Fig. 34. Average crosstalk distance for $10 \mu \mathrm{m}$ thick sapphire. 


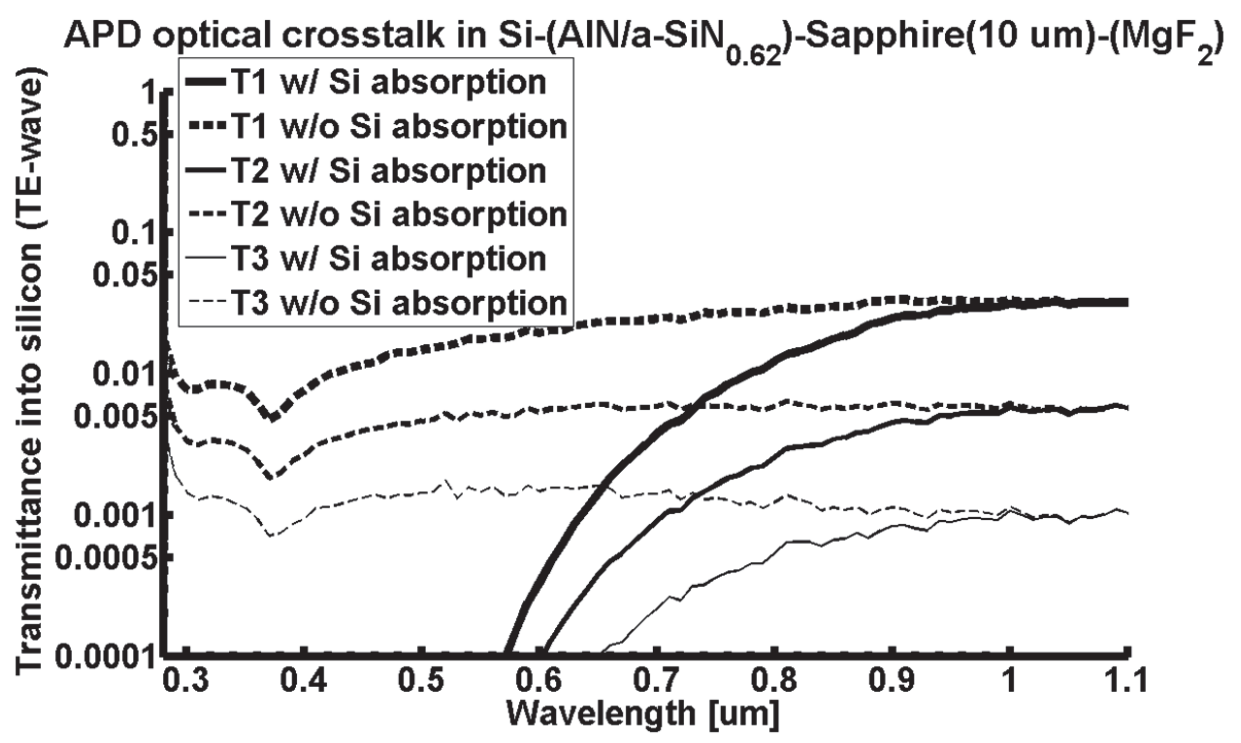

Fig. 35. Indirect APD optical crosstalk in $10 \mu \mathrm{m}$ thick sapphire.

The results in Fig. 34 show that the average distance of $T 1$ for a $10 \mu \mathrm{m}$ thick sapphire substrate corresponds to a crosstalk radius $C 1_{\mathrm{CT}} \approx 40 \mu \mathrm{m}$ of a circle fully inscribed into the square area formed by the eight adjacent pixels of the avalanching center APD shown in Fig. 30, where $C 1_{\mathrm{CT}}<C_{8-\mathrm{APDs}}=40.5 \mu \mathrm{m}$. Comparing the calculated results obtained in Sec. 3.1-3.2 for indirect optical crosstalk resulting from light emission during impact ionization in $27 \mu \mathrm{m}$ mesa APDs, respectively in Si-(AlN)-sapphire and $\mathrm{Si}$-(AlN/a-SiN 0.62$)$-sapphire substrates with back-side $\lambda / 4-\mathrm{MgF}_{2}$ antireflective layer, it is evident that the higher transmittance substrate with (AlN/a-SiN ${ }_{0.62}$ ) antireflective bilayer, also exhibits higher levels of indirect optical crosstalk. This result is expected since a larger fraction of light at points T1, T2 and T3 will be transmitted from sapphire into neighboring silicon mesa APDs due to the more efficient antireflective (AlN/a-SiN ${ }_{0.62}$ ) bilayer between sapphire and silicon compared to the $\lambda / 4$-AlN monolayer. In Sec. 3.3, a figure of merit is introduced for comparing the performance of the two different silicon-on-sapphire substrates analyzed in Sec. 3.1-3.2, based on the level of noise increase in the APD detector array resulting from indirect optical crosstalk from light emitted by the avalanche process. The results in Sec. 3.1-3.2 will be analyzed in Sec. 5 to assess their effect on the signal-to-noise ratio of the APD detectors in an array.

\subsection{Figure of merit for the noise performance of silicon-on-sapphire substrates due to} the APD emitted light

The results from the analysis of indirect optical crosstalk for $27 \mu \mathrm{m}$ mesa APDs fabricated in $\mathrm{Si}$-(AlN)-sapphire and $\mathrm{Si}$-(AlN/a-SiN 0.62$)$-sapphire substrates with $\lambda / 4-\mathrm{MgF}_{2}$ back-side 
antireflective layer in Sec. 3.1 and 3.2 respectively, show that the latter substrate with more efficient $\left(\mathrm{AlN} / \mathrm{a}-\mathrm{SiN}_{0.62}\right)$ antireflective bilayer between sapphire and silicon also produces greater levels of indirect optical crosstalk due to light emitted by the avalanche process. It is useful to be able to describe the levels of indirect optical crosstalk in $27 \mu \mathrm{m}$ mesa APD arrays using silicon-on-sapphire substrates from light emitted by the avalanche process, in terms of a figure of merit that allows comparison of the detector noise performance for the different back-illuminated substrates including Si-(AlN)-sapphire and Si-(AlN/a-SiN 0.62$)$-sapphire.

Fundamentally, optical crosstalk between closely spaced APD detectors in a high resolution array due to light emitted by the avalanche process, produces an increase in the detector noise in the array above the noise level of a standalone detector. To understand how the enhancement or increase in detector noise in an array occurs due to indirect optical crosstalk, it is helpful to consider the examples presented in Figs. 24 and 34, where indirect optical crosstalk from APD emitted light occurs primarily between an APD detector and its eight nearest neighbors, resulting from thinning of the sapphire substrate to $d_{\text {SAPPHIRE }}=10$ $\mu \mathrm{m}$. Assuming that the APDs are operating either in linear mode with gain or in non-linear Geiger-mode so that impact ionization and avalanche multiplication of charge carriers can occur, then the APD emitted photon flux resulting from impact ionization and avalanche gain will be given by Eq. (9). (Stern \& Cole, 2010)

$$
\Phi_{A P D 0}=\eta_{p}\langle G\rangle\left(T \eta_{a b s} \beta \Phi+\eta_{a} \Phi_{e}\right)
$$

In Eq. (9), $\Phi_{\mathrm{e}}$ describes the average number of thermally generated dark electrons per second and $T \eta_{\mathrm{abs}} \Phi$ describes the average number of photogenerated electrons per second where $T$ (shown in Fig. 13) represents the optical power transmittance into the device, $\eta_{\text {abs }}$ represents the absorption efficiency of light in the silicon and $\Phi$ represents the incident photon flux. In Eq. (9) it is assumed that both photogenerated and thermally generated electrons traversing the multiplication region of the APD produce secondary electrons through avalanche multiplication with an efficiency $\beta$ and $\eta_{\mathrm{a}}$ respectively. (Stern \& Cole, 2010) The electrons traversing the multiplication region of the APD produce photons with an efficiency $\eta_{\mathrm{P}}$ for each traversing electron. A higher average APD gain $<G>$ produces more photons since greater numbers of electrons traverse the multiplication region and the light generating efficiency $\eta_{\mathrm{P}}(E)$, depends on the electric field $E$, in the multiplication region which is greater at higher detector gain. The APD emitted photon flux in Eq. (9) has a wavelength range of $350<\lambda_{0}<1100 \mathrm{~nm}$ and therefore can be written as $\Phi_{\mathrm{APD}}(\lambda)$. (Akil et al., 1998, 1999)

In the $27 \mu \mathrm{m}$ mesa APD arrays analyzed in Secs. 3.1-3.2, the photons generated in the multiplication region and emitted isotropically, can only be transmitted to the eight nearest neighboring pixels through the wafer substrate. An increase in APD detector noise in an array occurs when a fraction of the APD emitted photon flux $\Phi_{\mathrm{APD} 0}$ from Eq. (9) is transmitted to the neighboring pixels, thereby increasing the multiplied electron flux $\left(T \eta_{\mathrm{abs}} \beta \Phi+\eta_{\mathrm{a}} \Phi_{\mathrm{e}}\right)$, in those devices that in turn increases their emitted photon flux $\Phi_{\mathrm{APD}}$, creating a positive feedback effect. The crosstalk generated multiplied electron flux is defined according to Eq. (10).

$$
\Phi_{C T 0}=T 1 \eta_{a b s} \beta \Phi_{A P D 0}
$$




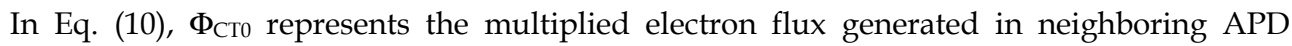
detectors as a result of the APD emitted photon flux $\Phi_{\text {APDo }}$ given by Eq. (9). The quantity $T 1$ >> T2 >> T3 was calculated in Sec. 3.1-3.2 for Si-(AlN)-sapphire and Si-(AlN/a-SiN $\mathrm{N}_{0.62}$ )sapphire substrates with $\lambda / 4-\mathrm{MgF}_{2}$ back-side antireflective layer and represents the fraction of the isotropically emitted APD light that is transmitted into neighboring APD detectors as shown in Figs. 23 and 33. Since the sapphire substrate $d_{\text {SAPPHIRE }}=10 \mu \mathrm{m}$, the multiplied electron flux $\Phi_{\text {Ст0 }}$ from Eq. (10) is produced in the eight adjacent detectors as shown in Figs. 20 and 30. The eight adjacent APD detectors however, each produce the same multiplied electron flux $\Phi_{\mathrm{CT} 0}$, in their respective eight adjacent pixels and therefore, the total multiplied electron flux in the APD will increase in a first approximation to $\left(T \eta_{\mathrm{abs}} \beta \Phi+\eta_{\mathrm{a}} \Phi_{\mathrm{e}}+\Phi_{\mathrm{CTO} 0}\right)$. Positive feedback will further increase $\Phi_{\mathrm{Cт}}$ and to calculate the increase, an indirect crosstalk parameter $D$ is defined according to Eq. (11).

$$
D=\frac{\Phi_{C T 0}}{T \eta_{a b s} \beta \Phi+\eta_{a} \Phi_{e}}=\eta_{p}\langle G\rangle T 1 \eta_{a b s} \beta
$$

The indirect optical crosstalk parameter $D$ in Eq. (11) represents the ratio between the multiplied electron flux generated in neighboring APD detectors as given by Eq. (10), with respect to the multiplied electron flux in the APD $\left(T \eta_{\mathrm{abs}} \beta \Phi+\eta_{\mathrm{a}} \Phi_{\mathrm{e}}\right)$, due to dark electrons and non-crosstalk, photogenerated electrons shown in Eq. (9). The indirect optical crosstalk parameter $D$, represents a useful figure of merit for the APD array design, describing the degree of indirect optical crosstalk that occurs through the substrate for different mean gain $<G>$ in the APD. The normal range of values for $D$ should be $0.0<D<1.0$. A lower $D$ value for a given mean gain $\langle G\rangle$, represents a higher performing substrate characterized by lower

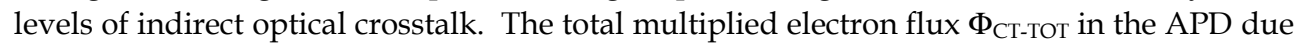
to indirect optical crosstalk can be calculated as shown in Eq. (12), using the indirect optical crosstalk parameter $D$.

$$
\Phi_{C T-T O T}=\sum_{k=0}^{\infty} \Phi_{C T 0} D_{k+1}^{k}=T 1 \eta_{a b s} \beta \Phi_{A P D 0}\left(1+D+D^{2}+\ldots+D^{k}\right)
$$

In Eq. (12), $k$ takes on integer values from 0 to $\infty$. It is evident from Eq. (12) that if the value of the indirect optical crosstalk parameter $D$, is between $0.0<D<1.0$, then $\Phi_{\text {С-тот }}$ converges, however, if $D>1$, then the noise current in the array will increase without bound. In practice, APD quench times in the Geiger-mode will limit the noise current growth, however, the imaging array will become dominated by noise and effectively rendered unusable. The total electron flux in an APD due to indirect optical crosstalk as given by Eq. (12), represents a mean value and should be independent of the distance of indirect optical crosstalk in the sapphire substrate, remaining valid whether the substrate has a thickness $d_{\text {SAPPHIRE }}=10$ or $50 \mu \mathrm{m}$.

The optical crosstalk parameter $D$ can be calculated using Eqs. (9-11), as a function of the mean detector gain $\langle G\rangle$, and different illumination conditions, for imaging arrays comprised of $27 \mu \mathrm{m}$ mesa APDs fabricated using Si-(AlN)-sapphire and Si-(AlN/a-SiN $\mathrm{N}_{0.62}$ )sapphire substrates with $\lambda / 4-\mathrm{MgF}_{2}$ back-side antireflective layer. The values of parameters used to calculate $D$ are given in Table 3 . 


$$
\begin{gathered}
\text { Parameter } \\
\text { Pixel size } \\
\text { Pixel area, } A_{\text {PIXEL }} \\
\text { Pixel height }
\end{gathered}
$$$$
\text { Focal plane array size }
$$$$
\text { FPA side length }
$$$$
\text { FPA area, } A_{\mathrm{FPA}}
$$

Camera lens focal length

Focal ratio setting, $f / \#$

Camera entrance aperture area, $A_{\mathrm{APERTURE}}$

Area of the sun's image projected onto the FPA, $A_{\text {SUN-FPA }}$

Total number of pixels that record the sun's projected image

APD focal plane array temperature

Photon generation efficiency in APD multiplication region
Value

$27 \mu \mathrm{m}$

$729 \times 10^{-8} \mathrm{~cm}^{2}$

$10 \mu \mathrm{m}$

$1024 \times 1024$

$2.7648 \mathrm{~cm}$

$7.644 \mathrm{~cm}^{2}$

$21 \mathrm{~cm}$

5.6

$11.04 \mathrm{~cm}^{2}$

$0.0309 \mathrm{~cm}^{2}$

4238 pixels

$T=243 \mathrm{~K}$

$\eta_{\mathrm{p}}=2.9 \times 10^{-5}$

Table 3. Indirect optical crosstalk calculation parameters.

The total unmultiplied electron flux due to photogenerated and dark electrons is calculated for the $27 \mu \mathrm{m}$ mesa APD in Figs. 36-37.

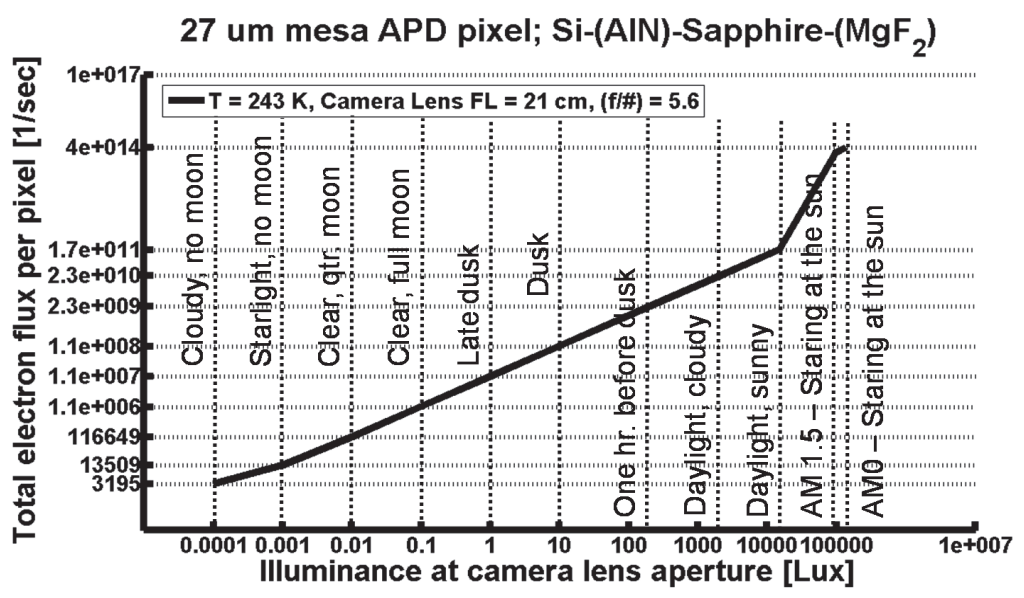

Fig. 36. Total unmultiplied electron flux $\left(T \eta_{\mathrm{abs}} \Phi+\Phi_{\mathrm{e}}\right)$ in APD. 


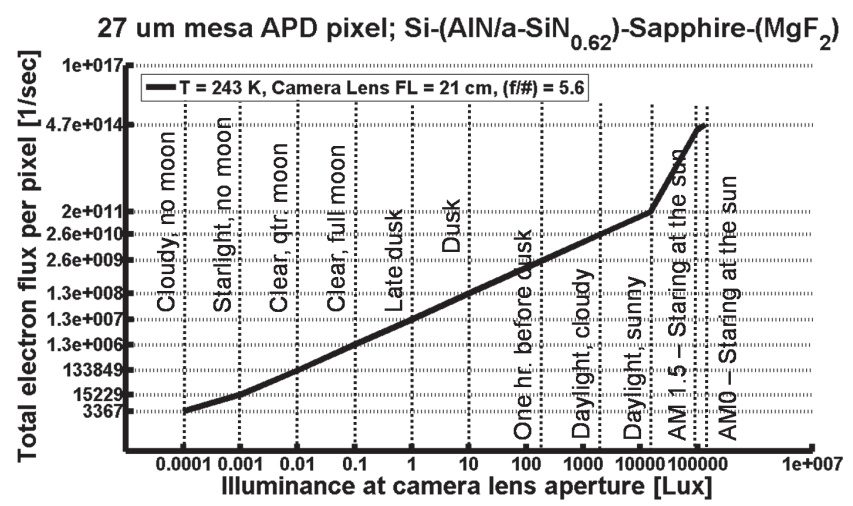

Fig. 37. Total unmultiplied electron flux $\left(T \eta_{\mathrm{abs}} \Phi+\Phi_{\mathrm{e}}\right)$ in APD.

In Figs. 36 and 37, the unmultiplied total electron flux in the $27 \mu \mathrm{m}$ mesa APD detector fabricated on $\mathrm{Si}-(\mathrm{AlN})$-sapphire or $\mathrm{Si}-\left(\mathrm{AlN} / \mathrm{a}-\mathrm{SiN}_{0.62}\right)$-sapphire with $\lambda / 4-\mathrm{MgF}_{2}$ back-side antireflective layer, is shown to increase as the illumination level at the camera lens increases. The camera lens has focal length $F=21 \mathrm{~cm}$ and an aperture stop setting $\mathrm{f} / \#=5.6$ as indicated in Table 3. The APD detector array operating temperature is set to $\mathrm{T}=243 \mathrm{~K}$ as provided by a two stage thermoelectric cooler. Using the results from Figs. 36-37 with Eqs. (9-11), the indirect optical crosstalk parameter $D$ for APD emitted light is calculated as a function of the average APD gain $\langle G\rangle$ in Fig. 38, for the lowest illumination condition occurring on a cloudy moonless night.

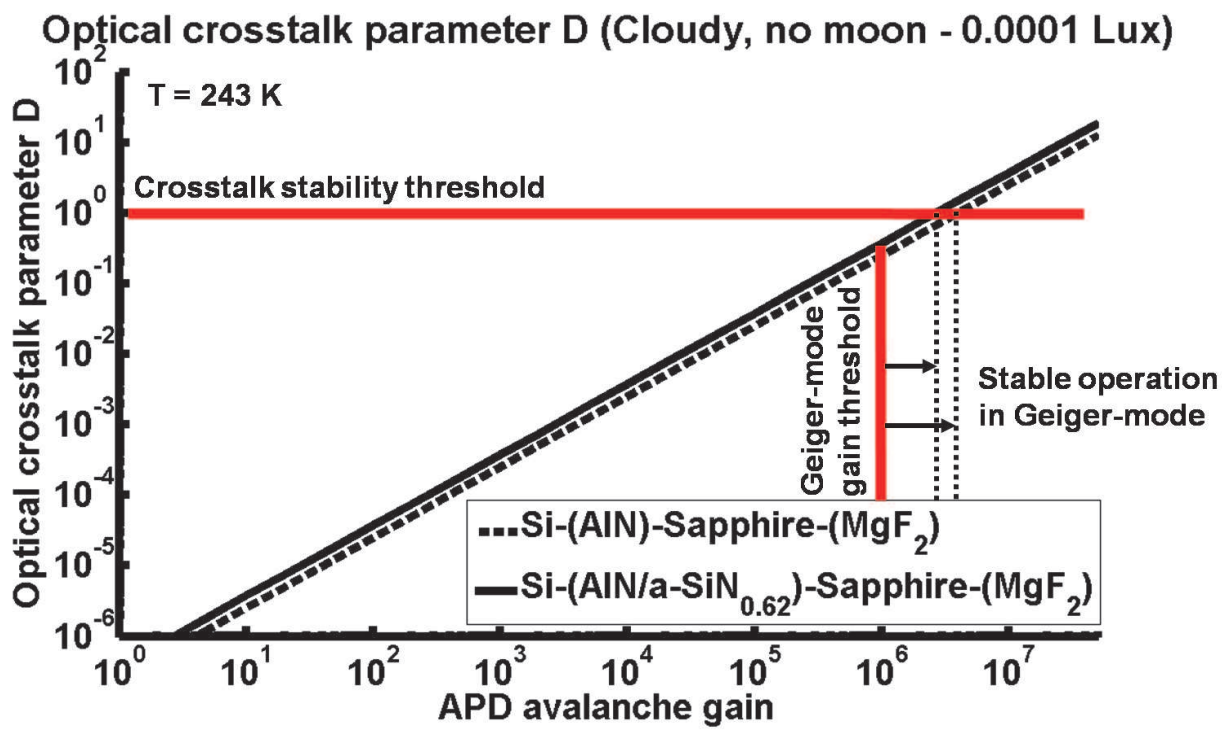

Fig. 38. Optical crosstalk parameter $D$ as a function of APD detector gain for the lowest natural illumination condition of 0.0001 lux at the camera lens, having focal length $F=21 \mathrm{~cm}$ and $f / \#=5.6$. 
The calculation in Fig. 38 considers a worst case example of crosstalk in the FPA, without silicon self-absorption of APD emitted light and approximates the spectral characteristic of the APD emitted photon flux $\Phi_{\mathrm{APD} 0}$ given by Eq. (9), as having a sharp emission peak at $2 \mathrm{eV}$ corresponding to $\lambda_{0}=620 \mathrm{~nm}$, rather than a broad emission spectrum of $350<\lambda_{0}<1100 \mathrm{~nm}$ described by Akil. The theory of Akil assumes that light emission below $2 \mathrm{eV}$ occurs due to indirect interband transitions, while bremsstrahlung generates the emission from $2.0-2.3 \mathrm{eV}$ and above $2.3 \mathrm{eV}$, direct interband transitions dominate, however, the theory does not consider light self-absorption in silicon. The theory of Lahbabi assumes an indirect interband recombination model and considers self-absorption of light in the silicon which for a multiplication region located at a height $h=9 \mu \mathrm{m}$ above the silicon-sapphire interface in the $27 \mu \mathrm{m}$ mesa APD, will absorb most of the UV and visible light as shown in Figs. 23 and 33, hence the transmission of mainly red light and NIR radiation into the sapphire substrate. Therefore, approximating that $\Phi_{\mathrm{APD} 0}$ given by Eq. (9) occurs at a monochromatic wavelength $\lambda_{0}=620 \mathrm{~nm}$ corresponding to a photon energy of $2 \mathrm{eV}$, is consistent with the results of Akil, Lahbabi and Rech, for the $27 \mu \mathrm{m}$ mesa APD design presented here. (Akil et al., 1998, 1999; Lahbabi et al., 2000; Rech et al., 2008)

The important result from Fig. 38 confirms that both Si-(AlN)-sapphire and Si-(AlN/a$\mathrm{SiN}_{0.62}$ )-sapphire wafer substrates with $\lambda / 4-\mathrm{MgF}_{2}$ back-side antireflective layer will support stable APD detector array operation at $\mathrm{T}=243 \mathrm{~K}$ in both the linear mode and Geiger-mode gain regimes, for the lowest levels of natural illumination of 0.0001 lux at the camera lens. The $27 \mu \mathrm{m}$ mesa APD detector must have an average gain $\langle G\rangle \leq 4 \times 10^{6}$ or $\langle G\rangle \leq 3 \times 10^{6}$ for $\mathrm{Si}$-(AlN)-sapphire and $\mathrm{Si}$-(AlN/a-SiN 0.62$)$-sapphire wafer substrates respectively, to preserve an optical crosstalk parameter $D<1$, necessary for stable array operation. Such a value of the gain is three times in magnitude above the commonly recognized $\langle G\rangle=1 \times 10^{6}$ gain threshold for Geiger-mode operation. The APD detector must therefore be designed and operated in a manner as to prevent the average gain from exceeding the limits for stable array operation. The result from Fig. 38 shows that the planar, high transmittance, backilluminated, silicon-on-sapphire wafer substrates described, will indeed support stable operation of high quantum efficiency and high resolution $27 \mu \mathrm{m}$ mesa APD detector arrays operating at the lowest level of natural illumination of 0.0001 lux at the camera lens in dual linear and Geiger-mode. Calculations in fact, confirm stable, wide dynamic range operation of the APD array over the full range of natural illumination conditions (shown in Figs. 3637) from AM 0 in space to the example in Fig. 38 of a cloudy moonless night. In Sec. 4, the indirect optical crosstalk from ambient incident illumination is calculated for the planar, back-illuminated, silicon-on-sapphire wafer substrates supporting high resolution, $27 \mu \mathrm{m}$ mesa APD detector arrays. The contribution of indirect optical crosstalk to the APD detector signal-to-noise ratio (SNR) will be analyzed in Sec. 5.

\section{Optical crosstalk from ambient light coupled into the sapphire waveguide}

It has been demonstrated in Sec. 3 that only a relatively small fraction of the photons generated by impact ionization in a $27 \mu \mathrm{m}$ mesa APD and emitted isotropically, are coupled into the planar sapphire substrate waveguide and transmitted to neighboring detectors, thereby contributing to an overall increase in noise levels in the array. In this section, a similar analysis considers indirect detector array optical crosstalk due to ambient light from 
a point source at infinity, incident on the back-illuminated, sapphire substrate waveguide undergoing multiple reflections and transmission into adjacent mesa APD detectors as shown in Fig. 15 and Fig. 39.

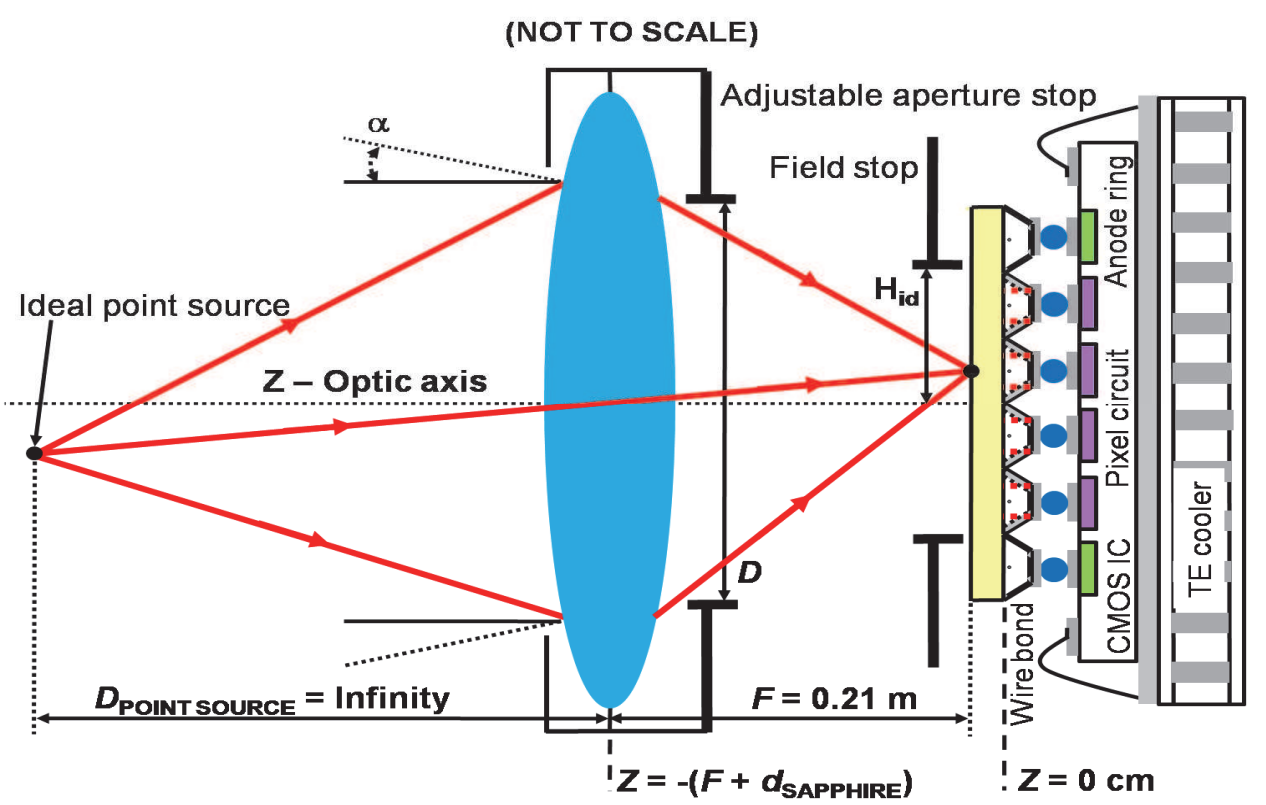

Fig. 39. Isotropic point source at infinity illuminates $27 \mu \mathrm{m}$ mesa APD in 1024x1024 FPA with $f / \#=5.6$ camera lens.

In Fig. 39, an ideal, isotropic point source of light is assumed to be located at an infinity distance, illuminating a $27 \mu \mathrm{m}$ mesa APD detector in a $1024 \times 1024$ pixel FPA through a camera lens with focal ratio setting $f / \#=5.6$. The camera lens and aperture stop or iris are circular, therefore, the Airy formula predicts a central disk or spot radius in the image plane for the ideal point source given approximately by Eq. (13).

$$
r_{\mathrm{SPOT}} \approx 1.22 \frac{F \lambda_{0}}{D}
$$

In Eq. (13), $F$ and $D$ are the camera lens focal length and diameter respectively and $\lambda_{0}$ is the optical wavelength given in micrometers. The diameter of the central Airy disk will therefore be approximately $5.6 \mu \mathrm{m}$ as calculated from Eq. (13) with $\lambda_{0}=0.41 \mu \mathrm{m}$ and $f / \#=$ 5.6, which is significantly smaller than the mesa APD detector pixel size of $27 \mu \mathrm{m}$. The subsequent analysis and calculation of indirect optical crosstalk will therefore assume that the point source of light at infinity is focused to an infinitesimal rather than a finite diameter point in the image plane, located directly at the center of the $27 \mu \mathrm{m}$ mesa APD base area as shown in Fig. 39. The optical k-vectors from the infinite distance point source of light arrive at various incidence angles at the image plane after focusing by the camera lens and are transmitted into the sapphire waveguide where they can undergo multiple reflections. 
A numerical or Monte Carlo simulation approach is used to calculate the fraction of light incident on a pixel which is transmitted to neighboring detectors by multiple reflections in the sapphire substrate. The simulation is performed on a $27 \mu \mathrm{m}$ mesa APD pixel located in the center of the $1024 \times 1024$ FPA, aligned with the optical axis of the imaging system shown in Fig. 39. Selecting the center pixel in the FPA for analysis as opposed to selecting a corner pixel, simplifies the indirect optical crosstalk calculation by ensuring that all of the optical kvectors emanating from the camera lens toward the center point of the pixel in the image plane are meridional rays contained in the same plane as the optical axis, hence there are no skew rays present. In a $1024 \times 1024$ APD-FPA with $27 \mu \mathrm{m}$ pixels and camera lens focal ratio $f / \#=5.6$ as shown in Fig. 39, the optical crosstalk from light incident at pixels near the corners of the FPA will be greater than for pixels near the optic axis, due to larger optical kvector incidence angles at corner pixels. The following Sec. 4.1-4.2, analyze and calculate the indirect optical crosstalk from ambient light incident on $27 \mu \mathrm{m}$ mesa APDs fabricated using respectively, $\mathrm{Si}$-(AlN)-sapphire and $\mathrm{Si}-\left(\mathrm{AlN} / \mathrm{a}-\mathrm{SiN}_{0.62}\right)$-sapphire substrates with $\lambda / 4-\mathrm{MgF}_{2}$ back-side antireflective layer.

\subsection{Indirect optical crosstalk from light incident on back-illuminated, sapphire waveguide; $\mathrm{Si}$-(AIN)-sapphire}

To study the nature of indirect optical crosstalk in APD-FPAs fabricated on planar, backilluminated, Si-(AIN)-sapphire substrates with $\lambda / 4-\mathrm{MgF}_{2}$ back-side antireflective layer without microlenses, due to incident illumination from a point source located at infinity as shown in Fig. 39, a Monte Carlo modeling and simulation approach is used. A 3-D Cartesian coordinate system can be defined where the z-axis represents the optic axis of the camera system as shown Fig. 39, and the camera lens with focal length $F=21 \mathrm{~cm}$ is located in the $\mathrm{x}-\mathrm{y}$ plane at $z=-\left(F+d_{\mathrm{SAPPHIRE}}+d_{\mathrm{AIN}}+d_{\mathrm{MgF} 2}\right)$. The $1024 \times 1024$ APD-FPA with $27 \mu \mathrm{m}$ mesa pixels is located at $z=0 \mathrm{~cm}$. Figure 40 shows a $3 \times 3$ array of $27 \mu \mathrm{m}$ mesa APD detectors and Fig. 41 shows the points of light transmittance T1, T2 and T3 in the sapphire substrate due to multiple reflections, for an optical k-vector incident to the $F=21 \mathrm{~cm}$ camera lens with focal ratio setting $f / \#=5.6$, from a point source located at infinity.

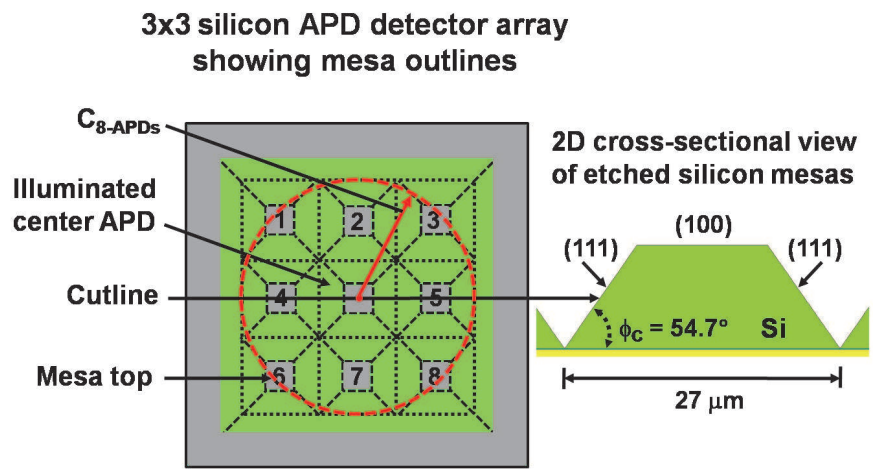

Fig. 40. 3×3 array showing eight immediately adjacent APDs. 


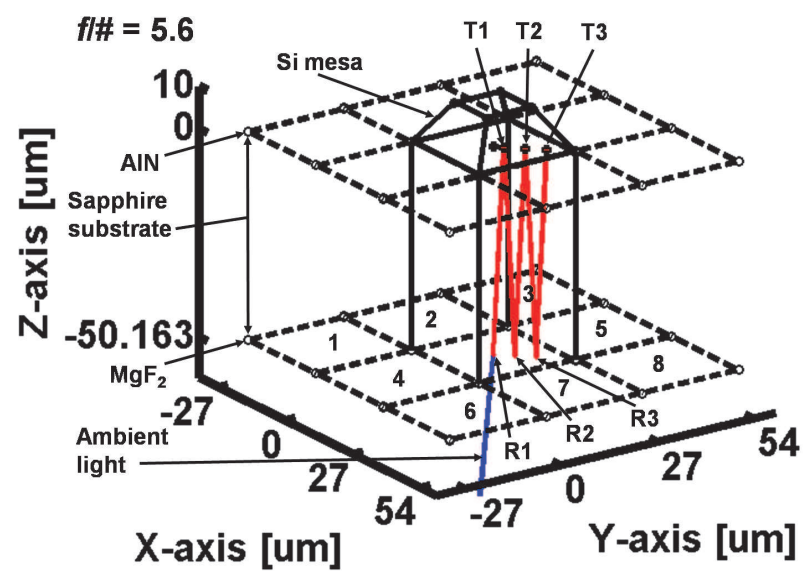

Fig. 41. 3-D ray tracing shows multiple reflections for $f / \#=5.6$.

In Fig. 42, 3-D ray tracing is used to calculate paths of light propagation for the randomly generated optical k-vectors from a point source at infinity over a $250<\lambda_{0}<1100 \mathrm{~nm}$ wavelength range, transmitted into the sapphire substrate and undergoing multiple reflections for a camera focal ratio $f / \#=5.6$. In Fig. 42 , even after three reflection cycles, the points of transmittance at $T 3$ occur inside the $27 \mu \mathrm{m}$ mesa pixel base area for the APD aligned with the camera optic axis and located in the center of the 1024x1024 FPA. Therefore, a camera focal ratio setting $f / \#=5.6$ produces negligible indirect optical crosstalk due to ambient incident light from a point source at infinity that is spatially conjugated to a $27 \mu \mathrm{m}$ mesa APD pixel aligned with the camera optic axis and located in the center of the 1024x1024 FPA. The results from Figs. 41-42, are used to calculate in Fig. 43, the fraction of the light incident at the APD aligned with the camera optic axis and located in the center of the $1024 \times 1024 \mathrm{FPA}$, that is transmitted at points $T 1, T 2$ and $T 3$ following reflections in the sapphire substrate, when the focal ratio setting $f / \#=5.6$.

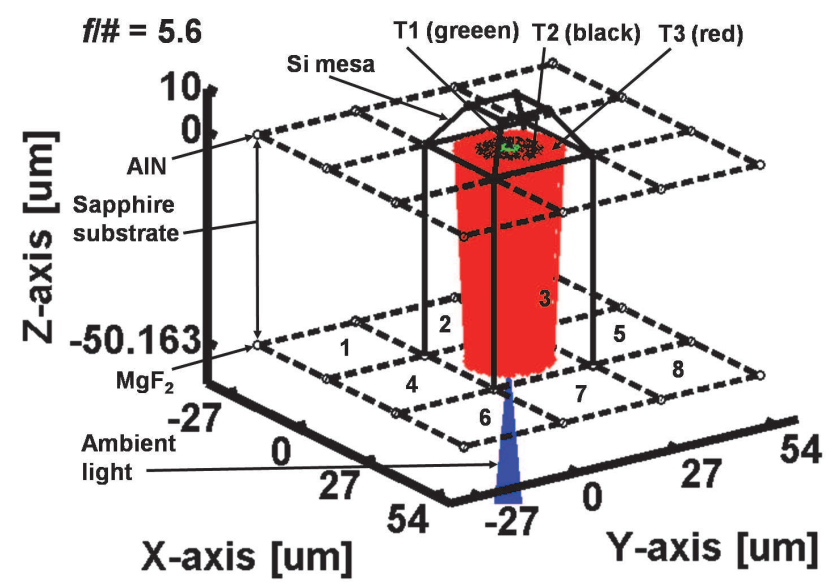

Fig. 42. 3-D ray tracing shows minimal crosstalk for $f / \#=5.6$. 


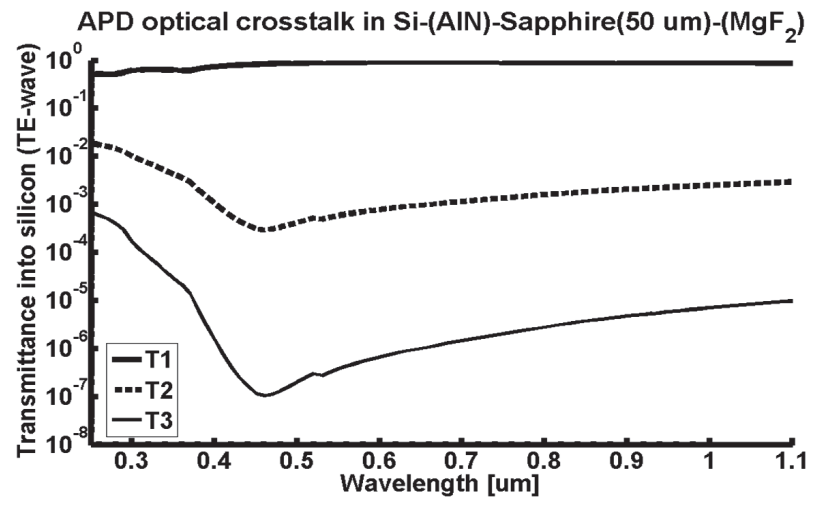

Fig. 43. Indirect crosstalk for $50 \mu \mathrm{m}$ thick sapphire and $f / \#=5.6$.

The Figs. 44-45 show light propagation paths for randomly generated optical k-vectors emitted by a point source at infinity over a $250<\lambda_{0}<1100 \mathrm{~nm}$ wavelength range, transmitted into the sapphire substrate and undergoing multiple reflections, for camera focal ratios $f / \#=16$ and $f / \#=2.0$ respectively.

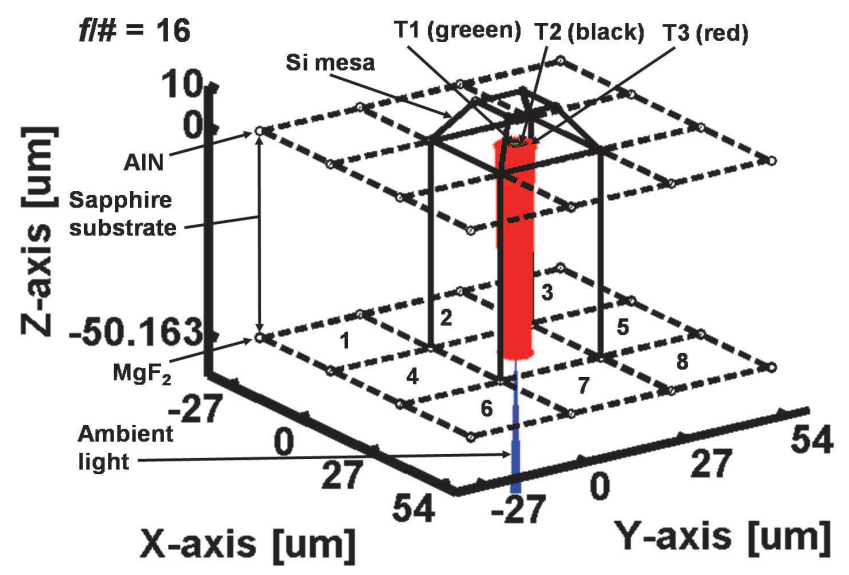

Fig. 44. 3-D ray tracing shows minimal crosstalk for $f / \#=16$. 


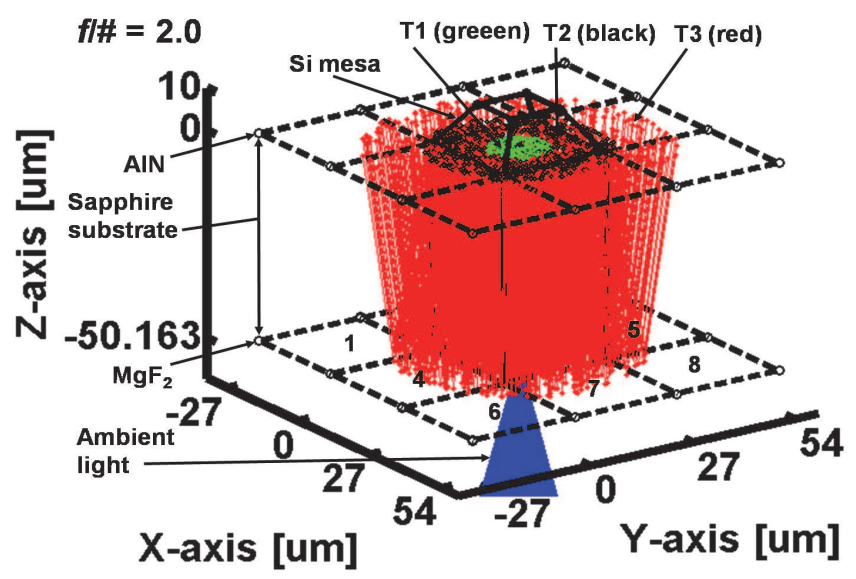

Fig. 45. 3-D ray tracing reveals indirect crosstalk for $f / \#=2.0$.

The indirect optical crosstalk due to incident illumination from a point source at infinity of a $27 \mu \mathrm{m}$ mesa APD pixel coincident with the camera optic axis and located in the center of the 1024x1024 FPA, has been shown to be negligible in planar Si-(AlN)-sapphire substrates with $\lambda / 4-\mathrm{MgF}_{2}$ back-side antireflective layer, $50 \mu \mathrm{m}$ thick sapphire and no microlenses. Although multiple reflections in the sapphire substrate occur for both the APD emitted light and the ambient incident illumination from a point source at infinity, the effect of the latter can be minimized by setting a higher camera focal ratio of $f / \#=5.6$ for example, to ensure that the multiple points of transmittance T1, T2 and T3 occur within the area of the illuminated 27 $\mu \mathrm{m}$ mesa APD. The same spatial confinement of multiply reflected optical k-vectors cannot be implemented as readily for the APD emitted light.

\subsection{Indirect optical crosstalk from light incident on back-illuminated, sapphire waveguide; $\mathrm{Si}$-(AIN/SiN $\left.\mathrm{S}_{0.62}\right)$-sapphire}

To study the nature of indirect optical crosstalk in APD-FPAs fabricated on planar, backilluminated, $\mathrm{Si}$-(AlN/a-SiN $\mathrm{Al}_{02}$ )-sapphire substrates with $\lambda / 4-\mathrm{MgF}_{2}$ back-side antireflective layer without microlenses, due to incident illumination from a point source located at infinity as shown in Fig. 39, a Monte Carlo modeling and simulation approach is used similar to Sec. 4.1. A 3-D Cartesian coordinate system can be defined where the z-axis represents the optic axis of the camera system as shown Fig. 39, and the camera lens with focal length $F=21 \mathrm{~cm}$ is located in the $x-y$ plane at $z=-\left(F+d_{\text {SAPPHIRE }}+d_{\mathrm{a}-\mathrm{SiN} \_0.62}+d_{\mathrm{AIN}}+\right.$ $d_{\mathrm{MgF} 2}$ ). The $1024 \times 1024$ APD-FPA with $27 \mu \mathrm{m}$ mesa pixels is located at $z=0 \mathrm{~cm}$. Figure 46 shows a $3 \times 3$ array of $27 \mu \mathrm{m}$ mesa APD detectors and Fig. 47 shows the points of light transmittance $T 1, T 2$ and $T 3$ in the sapphire substrate due to multiple reflections, for an optical k-vector incident to the $F=21 \mathrm{~cm}$ camera lens with focal ratio setting $f / \#=5.6$, from a point source located at infinity. 


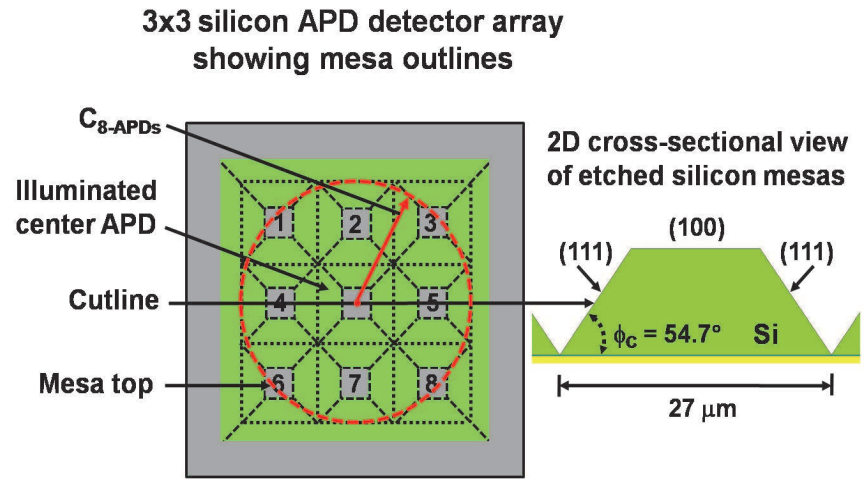

Fig. 46. $3 \times 3$ array showing eight immediately adjacent APDs.

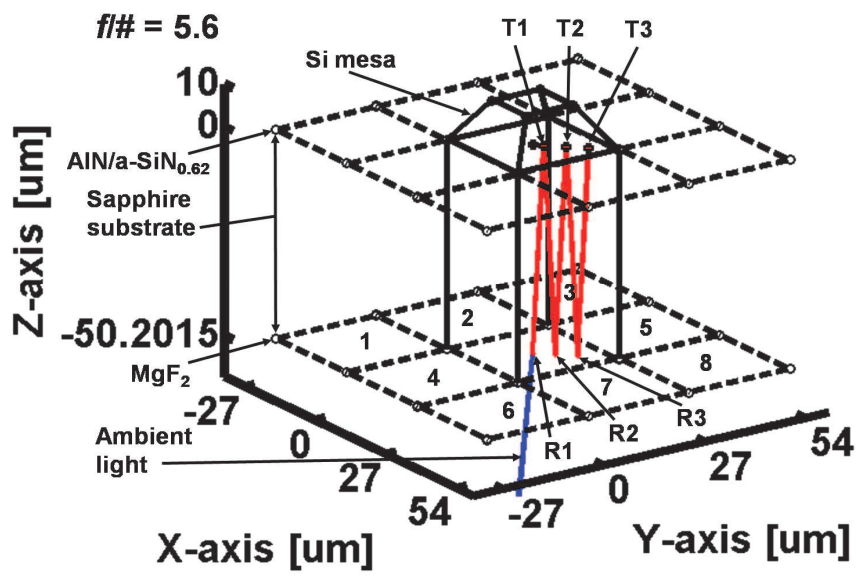

Fig. 47. 3-D ray tracing shows multiple reflections for $f / \#=5.6$.

In Fig. 48, 3-D ray tracing is used to calculate paths of light propagation for the randomly generated optical k-vectors from a point source at infinity over a $250<\lambda_{0}<1100 \mathrm{~nm}$ wavelength range, transmitted into the sapphire substrate and undergoing multiple reflections, for a camera focal ratio $f / \#=5.6$. In Fig. 48, even after three reflection cycles, the points of transmittance at $T 3$ occur inside the $27 \mu \mathrm{m}$ mesa pixel base area for the APD, which is aligned with the camera optic axis and located in the center of the 1024x1024 FPA. Therefore, a camera focal ratio setting $f / \#=5.6$ produces negligible indirect optical crosstalk due to ambient incident light from a point source at infinity, that is spatially conjugated to a $27 \mu \mathrm{m}$ mesa APD pixel aligned with the camera optic axis and located in the center of the 1024x1024 FPA. The results from Figs. 47-48, are used to calculate in Fig. 49, the fraction of the light incident at the APD aligned with the camera optic axis and located in the center of the $1024 \times 1024 \mathrm{FPA}$, that is transmitted at points $T 1, T 2$ and $T 3$ following reflections in the sapphire substrate, when the focal ratio setting $f / \#=5.6$. 


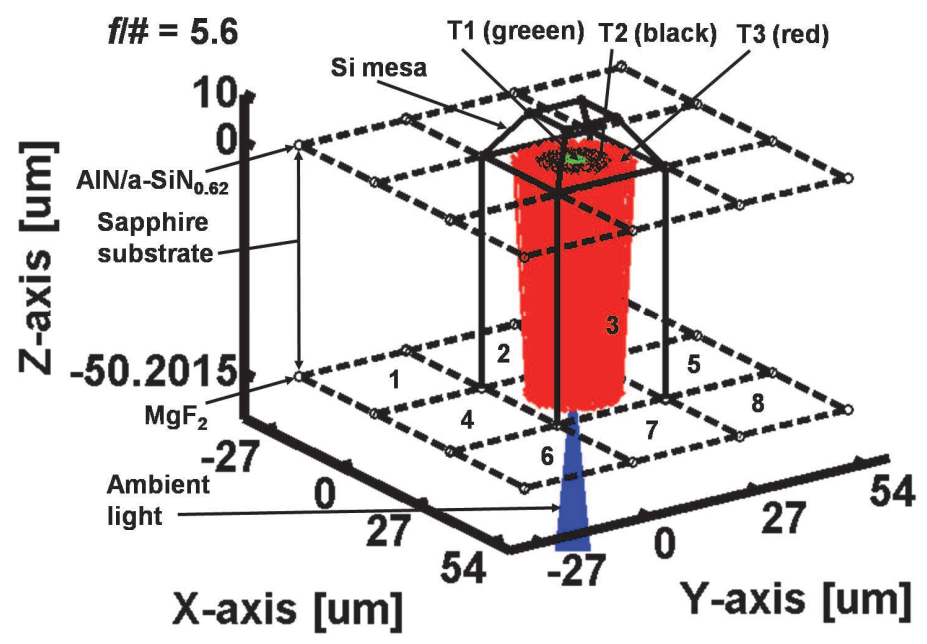

Fig. 48. 3-D ray tracing shows minimal crosstalk for $f / \#=5.6$.

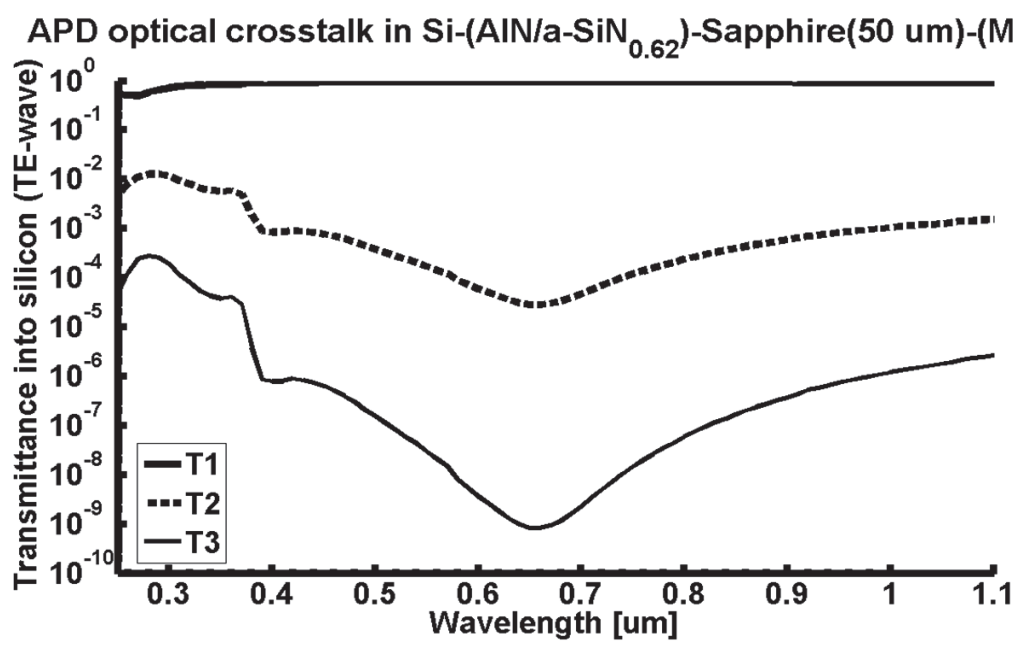

Fig. 49. Indirect crosstalk for $50 \mu \mathrm{m}$ thick sapphire and $f / \#=5.6$.

The Figs. 50-51 show light propagation paths for randomly generated optical k-vectors emitted by a point source at infinity over a $250<\lambda_{0}<1100 \mathrm{~nm}$ wavelength range, transmitted into the sapphire substrate and undergoing multiple reflections, for camera focal ratios $f / \#=16$ and $f / \#=2.0$ respectively. 


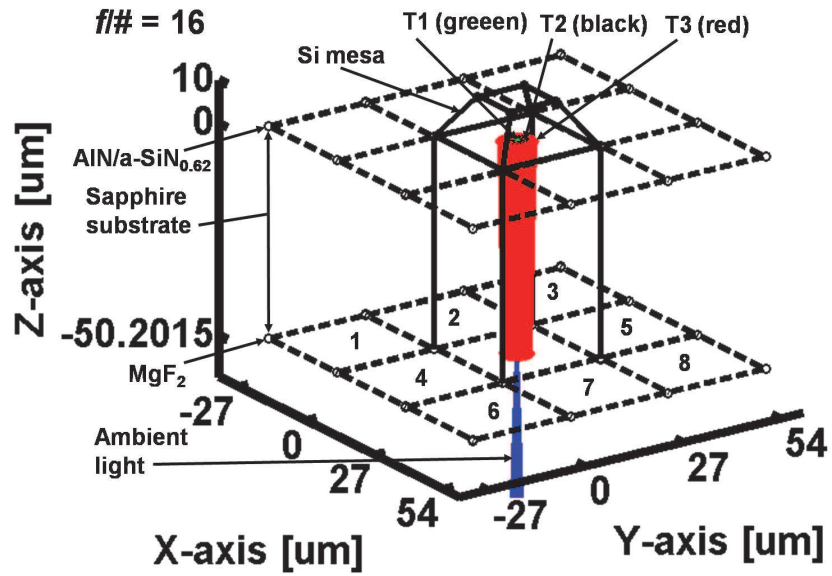

Fig. 50. 3-D ray tracing shows minimal crosstalk for $f / \#=16$.

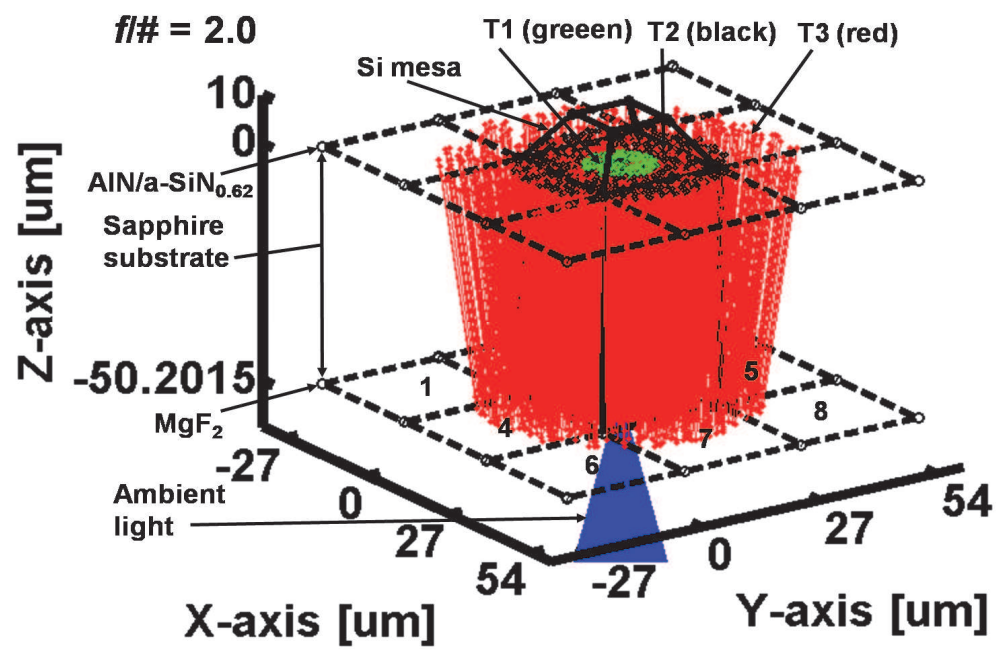

Fig. 51. 3-D ray tracing reveals indirect crosstalk for $f / \#=2.0$.

The focal ratio setting $f / \#=2.0$ in Fig. 51 produces some, although minimal indirect optical crosstalk due to multiple reflections in the sapphire substrate since the points of transmittance at $T 1$ occur inside the $27 \mu \mathrm{m}$ mesa pixel base area for the APD aligned with the camera optic axis and located in the center of the 1024x1024 FPA.

\section{Sensitivity of silicon-on-sapphire mesa APDs with indirect optical crosstalk}

The sensitivity or signal-to-noise ratio (SNR) of a back-illuminated, wide dynamic range $27 \mu \mathrm{m}$ mesa APD detector comprising a 1024x1024 pixel focal plane array (FPA), 
fabricated using $\mathrm{Si}-(\mathrm{AlN})$-sapphire or $\mathrm{Si}-\left(\mathrm{AlN} / \mathrm{a}-\mathrm{SiN}_{0.62}\right)$-sapphire substrates with $\lambda / 4$ $\mathrm{MgF}_{2}$ back-side antireflective layer, can be degraded by indirect optical crosstalk. Previous calculations have shown that the back-illuminated, $27 \mu \mathrm{m}$ mesa APD detector fabricated on Si-(AlN)-sapphire substrate with $\lambda / 4-\mathrm{MgF}_{2}$ back-side antireflective layer without a microlens and unaffected by optical crosstalk, will be capable of imaging with high sensitivity and wide dynamic range using dual linear and Geiger-mode, over the full range of natural illumination conditions from AM 0 in space to a cloudy moonless night. (Stern \& Cole, 2010) In this section, the sensitivity of the back-illuminated, $27 \mu \mathrm{m}$ mesa APD fabricated without a microlens, is calculated for the lowest level of natural illumination of 0.0001 lux at the camera lens, occurring on a cloudy moonless night. The sensitivity calculation considers the increased noise level in the detector due to indirect optical crosstalk from APD emitted light described in Sec. 3. The indirect optical crosstalk from ambient illumination of pixels described in Sec. 4, however, will not be considered in the detector sensitivity calculation due primarily to improved spatial confinement of optical k-vectors for $f / \# \geq 5.6$, compared to the APD emitted light in Sec. 3. The expressions in Eqs. (14-15) yield the wide dynamic range, $27 \mu \mathrm{m}$ mesa APD detector signal-to-noise ratio with optical crosstalk for the linear and Geiger-mode optical receivers respectively, assuming the optical crosstalk current is independent from the detector photocurrent and dark current.

$$
\begin{gathered}
S N R_{A P D-\text { linear-receiver }}=\frac{\left\langle i_{p}(t)\right\rangle^{2}}{\sigma_{i_{p}}^{2}+\sigma_{i_{D}}^{2}+\sigma_{i_{C T}}^{2}+\sigma_{i_{e n}}^{2}} \\
S N R_{A P D-\text { Geiger-receiver }}=\frac{\left\langle i_{p}(t)\right\rangle^{2}}{\sigma_{i_{p}}^{2}+\sigma_{i_{D}}^{2}+\sigma_{i_{C T}}^{2}}
\end{gathered}
$$

In Eqs. (14-15), the signal is given by the square of the mean detector photocurrent $i_{\mathrm{p}}(\mathrm{t})$, and the noise is given by the sum of the variances of the photocurrent, dark current, optical crosstalk current and electronic readout circuit current, which are all assumed to be independent. The signal-to-noise ratios given by Eqs. (14-15) were previously calculated by Stern for the wide dynamic range $27 \mu \mathrm{m}$ mesa APD without a microlens using $\mathrm{Si}$-(AIN)-sapphire substrate with $\lambda / 4-\mathrm{MgF}_{2}$ back-side antireflective layer in the absence of optical crosstalk. (Stern \& Cole, 2010) The expressions for the photoelectron current variance, dark current variance and electronic readout circuit noise were also derived. (Stern \& Cole, 2010) After late dusk, the wide dynamic range $27 \mu \mathrm{m}$ mesa APD can be operated in the non-linear Geiger-mode without saturating the maximum count rate of the detector, for a 50 ns quench time. (Kumar et al., 2004) In the Geiger-mode, the optical receiver sensitivity is calculated according to Eq. (15) where the contribution of the electronic circuit noise has been eliminated by direct photon-to-digital conversion in the detector pixel. (Ghioni et al., 1996)

In Eqs. (14-15), it is assumed that the indirect optical crosstalk current due to APD emitted light $i_{\mathrm{CT}}$ is independent from the photocurrent $i_{\mathrm{p}}$ and from the dark current $i_{\mathrm{D}}$ in the $27 \mu \mathrm{m}$ mesa APD detector, thereby allowing the variances $\sigma_{\mathrm{i}_{-}} \mathrm{p}^{2}, \sigma_{\mathrm{i}_{-} \mathrm{D}}{ }^{2}$ and $\sigma_{\mathrm{i}_{-} \mathrm{CT}}{ }^{2}$ to be added as 
shown. The assumption of independence for $i_{\mathrm{p}}, i_{\mathrm{D}}$ and $i_{\mathrm{CT}}$ in the $27 \mu \mathrm{m}$ mesa APD detector

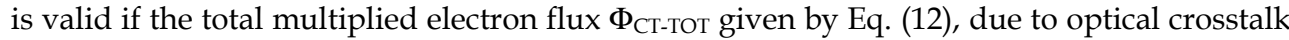
from the neighboring pixels is given by the sum of even $k$ terms $(k=0,2,4 \ldots)$. The variance of the crosstalk current $\sigma_{\mathrm{i}} \mathrm{CT}^{2}$ in Eqs. (14-15), due solely to even $k$ term contribution to the total multiplied crosstalk electron flux $\Phi_{\mathrm{CT}-\mathrm{TOT}}$, has a similar form to the photocurrent variance $\sigma_{\mathrm{i}} \mathrm{p}^{2}$. (Stern \& Cole, 2010) The inclusion of odd higher order $k$ terms $(k=1,3,5 \ldots)$ in calculating Eq. (12), would require Eqs. (14-15) to be modified, to account for correlations between the photoelectron current and dark current with the crosstalk current in the $27 \mu \mathrm{m}$ mesa APD. Figure 52 shows the SNR of $27 \mu \mathrm{m}$ mesa APD detectors fabricated on Si-(AlN)sapphire and $\mathrm{Si}$-(AlN/a-SiN 0.62$)$-sapphire substrates with $\lambda / 4-\mathrm{MgF}_{2}$ back-side antireflective layer, calculated according to Eq. (15), using a fixed Geiger-mode gain $\langle G\rangle=1 \times 10^{6}$ and including only the $k=0$ term also given by Eq. (10), for calculating the total multiplied

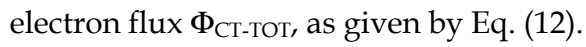

\section{Optical receiver SNR vs. $T_{\text {INT }}$ (Cloudy, no moon - 0.0001 Lux)}

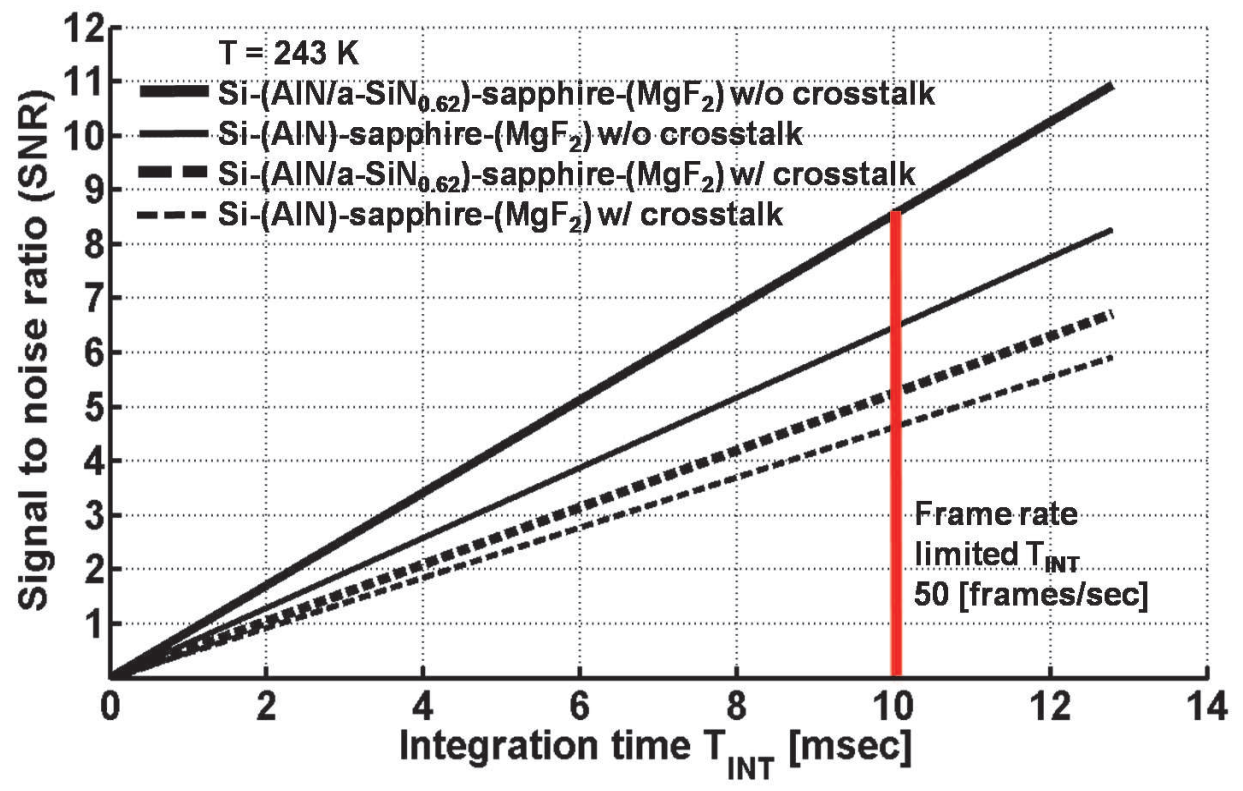

Fig. 52. SNR of the Geiger-mode APD imager on a cloudy moonless night and 0.0001 lux illumination. The Geiger-mode APD has 8-bit resolution and a quench time of $50 \mathrm{~ns}$.

It is evident in Fig. 52, that indirect optical crosstalk in back-illuminated, high resolution, 27 $\mu \mathrm{m}$ mesa APD arrays fabricated on $\mathrm{Si}-(\mathrm{AlN})$-sapphire and $\mathrm{Si}$-(AlN/a-SiN 0.62$)$-sapphire substrates with $\lambda / 4-\mathrm{MgF}_{2}$ back-side antireflective layer without microlenses, reduces the detector sensitivity from $\mathrm{SNR} \approx 6$ to $\mathrm{SNR} \approx 4$ and from $\mathrm{SNR} \approx 8$ to $\mathrm{SNR} \approx 5$ respectively. Despite indirect optical crosstalk, the Geiger-mode APD-FPA will be capable of imaging at 
the lowest level of natural illumination of 0.0001 lux at the camera lens at 50 frames per second with 8-bit resolution.

\section{Conclusion}

The detailed analysis in this chapter has confirmed an important result, namely, that the sensitivities of the $27 \mu \mathrm{m}$ mesa APD detectors comprising back-illuminated, silicon-onsapphire FPAs without monolithic microlenses, are not degraded by indirect optical crosstalk phenomena to the level that would prevent high sensitivity imaging, even for the highest (Geiger-mode) gain regime of operation. Thus, for all but the most demanding imaging applications, monolithic sapphire microlenses are not required. This result is important because sapphire microlens fabrication and in general any fabrication step adds to the complexity and cost of manufacturing. The calculations and analysis in this chapter have referred primarily to silicon APD detector arrays fabricated using novel, backilluminated silicon-on-sapphire substrates, however, the substrate technology will inherently support not only silicon, but also epitaxially grown $\mathrm{Si}_{(1-x)} \mathrm{Gex}_{\mathrm{X}}$ APD detector arrays. The family of high transmittance silicon-on-sapphire substrates represents a key enabling technology for the next generation of ultrasensitive, solid-state, high quantum efficiency and high resolution avalanche detector arrays. As the novel substrate technology is developed commercially, new as yet to be defined and designed imager concepts will emerge with it.

\section{References}

Akil, N., Kerns, S.E., Kerns, D.V., Hoffmann, A. \& Charles, J.P. (1998). "Photon generation by silicon diodes in avalanche breakdown," Applied Physics Letters, 73(7), 871.

Akil, N., Kerns, S.E., Kerns, D.V., Hoffmann, A. \& Charles, J.P. (1999). “A Multimechanism Model for Photon Generation by Silicon Junctions in Avalanche Breakdown," IEEE Transactions on Electron Devices, 46(5), 1022-1028.

Charbon, E. (2008). “Towards large scale CMOS single-photon detector arrays for lab-onchip applications," Journal of Physics D: Applied Physics, 41(9), 4010.

Gardeniers, J.G.E., Tilmans, H.A.C. \& Visser, C.C.G. (1996). “LPCVD silicon-rich silicon nitride films for applications in micromechanics, studied with statistical experimental design," Journal of Vacuum Science Technology A, 14(5), 28792892.

Ghioni, M., Cova, S., Zappa, F. \& Samori, C. (1996). “Compact active quenching circuit for fast counting with avalanche photodiodes," Review of Scientific Instruments, 67(10), 3440-3448.

Guerrieri, F., Tisa, S. \& Zappa, F. (2009). “Fast Single-Photon Imager acquires 1024 pixels at 100 kframes/s," Proc. of SPIE Vol. 7249, 72490U.

Imthurn, G.P., Garcia, G.A., Walker, H.W. \& Forbes, L. (1992). “Bonded silicon-on-sapphire wafers and devices," Journal of Applied Physics, 72(6), 2526-2527. 
Kumar, P., Kwiat, P., Migdall, A., Nam, S.W., Vuckovic, J. \& Wong, N.C. (2004). “Photonic Technologies for Quantum Information Processing," Quantum Information Processing, 3(1-5), 215-231.

Lahbabi, M., Ahaitouf, A., Abarkan, E., Fliyou, M., Hoffmann, A., Charles, J.P., Bhuva, B.L., Kerns, S.E. \& Kerns, D.V. (2000). “Analysis of electroluminescence spectra of silicon junctions in avalanche breakdown using an indirect interband recombination model," Applied Physics Letters, 77(20), 3182.

Lau, S.S., Matteson, S., Mayer, J.W., Revesz, P. \& Gyulai, J. (1979). "Improvement of Crystalline Quality of Epitaxial Silicon Layers by Ion-Implantation Techniques," Applied Physics Letters, 34(1), 76.

Lowe, A.J., Powell, M.J. \& Elliot, S.R. (1986). “The electronic properties of plasma deposited films of hydrogenated amorphous $\operatorname{SiN}_{X}(0<x<1.2)$," Journal of Applied Physics, 59(4), 1251.

Makino, T. (1983). "Composition and Structure Control by Source Gas Ratio in LPCVD $\mathrm{SiN}_{\mathrm{X}}$," Journal of Electrochemical Society, 130(2), 450-455.

Manasevit, H.M. \& Simpson, W.J. (1964). “Single-Crystal Silicon on a Sapphire Substrate," J. of Appl. Phys., 35(4), 1349-1351.

Niclass, C., Rochas, A., Besse, P.A., Popovic, R.S. \& Charbon, E. (2005). “CMOS Imager Based on Single Photon Avalanche Diodes," IEEE 13th Internl. Conference on Solid-State Sensors, Actuators and Microsystems, Seoul, S. Korea, 1, 10301034.

Park, S.H., Jeon, H., Sung, Y.J. \& Yeom, G.Y. (2001). “Refractive sapphire microlenses fabricated by chlorine-based inductively coupled plasma etching," Applied Optics, 40(22), 3698-3702.

Plummer, J.D., Deal, M.D. \& Griffin, P.D. (2000). [Silicon VLSI Technology], Prentice Hall, Upper Saddle River NJ.

Rech, I., Ingargiola, A., Spinelli, R., Labanca, I., Marangoni, S., Ghioni, M. \& Cova, S. (2008). "A New Approach to Optical Crosstalk Modelling in Single-Photon Avalanche Diodes," IEEE Photonics Technology Letters, 20(5), 330-332.

Rosler, R.S. (1977). "Low Pressure CVD Production Processes for Poly, Nitride and Oxide," Solid State Technology, 20(4), 63.

Saleh, B.E.A. \& Teich, M.C. (2007). [Fundamentals of Photonics], John Wiley and Sons, New York, 246-253, 755, 789.

Stern, A.G. \& Cole, D.C. (2010). "High-sensitivity, wide-dynamic-range avalanche photodiode pixel design for large-scale imaging arrays," Journal of Electronic Imaging, Special Section on Digital Photography, 19(2).

Stern, A.G. \& Cole, D.C. (2008). "Design of a back-illuminated, crystallographically etched, silicon-on-sapphire avalanche photodiode with monolithically integrated microlens, for dual-mode passive \& active imaging arrays," Proc. of SPIE Vol. 7153, $71530 \mathrm{Z}$.

Stern, A.G. et al. (2003). “Intensity-imaging focal plane array using Geiger-mode avalanche photodiodes," Solid State Research Report MIT Lincoln Laboratory, (4), 23-31. 
Sze, S.M. (1981). [Physics of Semiconductor Devices 2nd Edition], John Wiley \& Sons, New York.

Tauc, J. (1974). [Amorphous and Liquid Semiconductors], Plenum Press, New York, 173.

Verlaan, V., Bakker, R., van der Werf, C.H.M., Houweling, Z.S., Mai, Y., Rath, J.K. \& Schropp, R.E.I. (2007). "High-density silicon nitride deposited at low substrate temperature with high deposition rate using hot wire chemical vapour deposition," Surface \& Coatings Technology, 201, 9285-9288. 


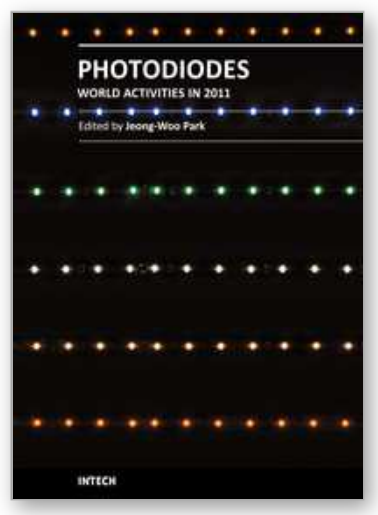

\author{
Photodiodes - World Activities in 2011 \\ Edited by Prof. Jeong Woo Park
}

ISBN 978-953-307-530-3

Hard cover, 400 pages

Publisher InTech

Published online 29, July, 2011

Published in print edition July, 2011

Photodiodes or photodetectors are in one boat with our human race. Efforts of people in related fields are contained in this book. This book would be valuable to those who want to obtain knowledge and inspiration in the related area.

\title{
How to reference
}

In order to correctly reference this scholarly work, feel free to copy and paste the following:

Alvin G. Stern (2011). Design of High Quantum Efficiency and High Resolution, Si/SiGe Avalanche Photodiode Focal Plane Arrays Using Novel, Back-Illuminated, Silicon-on-Sapphire Substrates, Photodiodes - World Activities in 2011, Prof. Jeong Woo Park (Ed.), ISBN: 978-953-307-530-3, InTech, Available from: http://www.intechopen.com/books/photodiodes-world-activities-in-2011/design-of-high-quantum-efficiency-andhigh-resolution-si-sige-avalanche-photodiode-focal-plane-array

\section{INTECH}

open science | open minds

\author{
InTech Europe \\ University Campus STeP Ri \\ Slavka Krautzeka 83/A \\ 51000 Rijeka, Croatia \\ Phone: +385 (51) 770447 \\ Fax: +385 (51) 686166 \\ www.intechopen.com
}

\author{
InTech China \\ Unit 405, Office Block, Hotel Equatorial Shanghai \\ No.65, Yan An Road (West), Shanghai, 200040, China \\ 中国上海市延安西路65号上海国际贵都大饭店办公楼405单元 \\ Phone: +86-21-62489820 \\ Fax: +86-21-62489821
}


(C) 2011 The Author(s). Licensee IntechOpen. This chapter is distributed under the terms of the Creative Commons Attribution-NonCommercialShareAlike-3.0 License, which permits use, distribution and reproduction for non-commercial purposes, provided the original is properly cited and derivative works building on this content are distributed under the same license. 